\title{
Hepatocyte CREBZF-osteopontin axis controls fibrosis in nonalcoholic steatohepatitis
}

\section{Fengguang Ma}

Shanghai Institute of Nutrition and Health, SIBS, CAS

Yuxiao Liu

Shanghai Institute of Nutrition and Health, SIBS, CAS

Zhimin Hu

Institute for Nutritional Sciences, Shanghai Institutes for Biological Sciences, Chinese Academy of

Sciences

\section{Yaqian Xue}

Shanghai Institute of Nutrition and Health, SIBS, CAS

\section{Zhengshuai Liu}

Shanghai Institute of Nutrition and Health, SIBS, CAS

\section{Genxiang Cai}

Shanghai Institute of Nutrition and Health, SIBS, CAS

\section{Weitong Su}

Shanghai Institute of Nutrition and Health, SIBS, CAS

\section{Zengpeng Zheng}

Shanghai Institute of Nutrition and Health, SIBS, CAS

\section{Dong Ding}

Shanghai Institute of Nutrition and Health, SIBS, CAS

\section{Xiaoyang Sun}

Zhongshan Hospital, Fudan University

\section{Yang Jiang}

Tianjin University of Science and Technology

\section{Shuang Wei}

Shanghai Institute of Nutrition and Health, SIBS, CAS

\section{Wenjing Li}

Shanghai Institute of Nutrition and Health, SIBS, CAS

\section{Yong Xu}

The Affiliated Hospital of Southwest Medical University

\section{Dongguang Xiao}

Tianjin University of Science and Technology

\section{Cuiying Zhang}

Tianjin University of Science and Technology 


\section{Xin Gao}

Zhongshan Hospital, Fudan University

\section{Xiaozhen Dai}

University of Louisville

\section{Yu Li ( $\sim$ liyu@sibs.ac.cn )}

Shanghai Institute of Nutrition and Health, SIBS, CAS https://orcid.org/0000-0001-6910-5933

\section{Aoyuan Cui}

Institute of Nutrition and Health, Chinese Academy of Sciences

\section{Article}

Keywords: CREBZF, miR-6964-3p, osteopontin, fibrosis, NASH

Posted Date: September 29th, 2021

DOI: https://doi.org/10.21203/rs.3.rs-929976/v1

License: (c) (1) This work is licensed under a Creative Commons Attribution 4.0 International License.

Read Full License 


\section{Hepatocyte CREBZF-osteopontin axis controls fibrosis in nonalcoholic steatohepatitis}

Fengguang Ma ${ }^{1}$, Yuxiao Liu${ }^{1}$, Zhimin Hu${ }^{1}$, Yaqian Xue ${ }^{1}$, Zhengshuai Liu ${ }^{1}$, Genxiang Cai ${ }^{1}$, Weitong Su${ }^{1}$, Zengpeng Zheng ${ }^{1}$, Dong Ding ${ }^{1}$, Xiaoyang Sun ${ }^{2}$, Yang Jiang ${ }^{3}$, Shuang $\mathrm{Wei}^{1}$, Wenjing $\mathrm{Li}^{1}$, Yong $\mathrm{Xu}^{4}$, Dongguang $\mathrm{Xiao}^{3}$, Cuiying Zhang ${ }^{3}$, Xin $\mathrm{GaO}^{2}$, Xiaozhen Dai ${ }^{5}$, Yu Li ${ }^{1 *}$, Aoyuan Cui ${ }^{* *}$

${ }^{1}$ CAS Key Laboratory of Nutrition, Metabolism and Food Safety, Shanghai Institute of Nutrition and Health, University of Chinese Academy of Sciences, Chinese Academy of Sciences, Shanghai 200031, China; 2Department of Endocrinology and Metabolism, Zhongshan Hospital, Fudan University, Shanghai 200032, China; ${ }^{3}$ College of Biotechnology, Tianjin University of Science and Technology, Tianjin 300457, China; ${ }^{4}$ Department of Endocrinology and Metabolism, The Affiliated Hospital of Southwest Medical University, Luzhou, Sichuan 646000, China; ${ }^{5}$ School of Bioscience and Technology, Chengdu Medical College, Chengdu, Sichuan 610500, China;

\section{Running Title: CREBZF-osteopontin axis promotes fibrosis in NASH}

* Corresponding Author:

Yu Li, PhD

Professor

Shanghai Institute of Nutrition and Health

Chinese Academy of Sciences

320 Yue Yang Road

Life Science Research Building A1816

Shanghai 200031, China

Phone: +86 (21) 54920753

Email: liyu@sibs.ac.cn

Aoyuan Cui, PhD

Research Associate

Shanghai Institute of Nutrition and Health

Chinese Academy of Sciences

320 Yue Yang Road

Life Science Research Building A1818

Shanghai 200031, China

Phone: +86 (21) 54920924

Email: aycui@sibs.ac.cn

Funding: This work was supported by grants from National Key R\&D Program of China (2019YFA0802502), National Natural Science Foundation of China (81925008), Key Laboratory of Wuliangye-flavor Liquor Solid-state Fermentation of China National Light Industry (2019JJ005), Shanghai Science and Technology Commission (19140903300) to Y.L. This work was also supported by grants from China Postdoctoral Science Foundation (2021M693273) to A.C.

\section{Author Contribution:}

F.M., A.C. and Y.L. contributed to experiment design; F.M., Y.Liu., Z.H., Y.X., Z.L., G.C., W.S., Z.Z, D.D., X.S., Y.J., S.W., W.L., X.D., A.C. contributed to the acquisition and analysis of data; and Y.X, D.X., C.Z., X.G. reviewed the manuscript; Y.L. and A.C. obtained the funding; F.M., A.C. and Y.L. wrote the manuscript.

\section{The authors disclose no conflicts of interest}




\begin{abstract}
Nonalcoholic steatohepatitis (NASH) has emerged as a leading cause of chronic liver disease. The incomplete understanding of NASH fibrosis limits pharmacotherapy development. Here we report a molecular link between hepatocytes and hepatic stellate cells (HSCs) in regulating the progression of liver fibrosis via CREBZF-osteopontin (OPN) axis. Hepatocyte-specific CREBZF knockout (CREBZF LKO) mice and their wild-type littermates were divided into groups that were placed on AMLN, MCD or chow diet. Mouse primary hepatocytes were treated with $250 \mu \mathrm{M} \mathrm{PA}$ and $10 \mathrm{ng} / \mathrm{ml} \mathrm{TNFa}$, the conditioned medium was collected and then transferred to HSC-T6 cells for 24 hours. Adeno-associated virusmediated overexpression of OPN or CREBZF was performed in mice via tail vail injection. Human studies have shown that deregulation of CREBZF is associated with pathogenesis of hepatic steatosis and dyslipidemia. Here, we show that CREBZF is markedly elevated in livers of NASH mice, whereas hepatocyte-specific CREBZF knockout mice are prevented from AMLN or MCD diet-induced hepatic inflammation, liver injury and fibrosis. In vivo and in vitro mechanistic studies revealed that a key mechanism linking hepatocyte CREBZF to NASH fibrosis is miR-6964-3p-mediated inhibition of OPN, an extracellular matrix protein that activates fibrogenic genes in hepatic stellate cells. Moreover, the reduction of NASH phenotypes in CREBZF LKO mice was reversed by adeno-associated virus-mediated overexpression of OPN. Thus, CREBZF-OPN axis represents a previously unrecognized intrahepatic crosstalk in the liver that triggers fibrosis progression and contributes to the severity of NASH.
\end{abstract}

Keywords: CREBZF, miR-6964-3p, osteopontin, fibrosis, NASH

\title{
Highlights
}

- Hepatic CREBZF is required for NASH diet-induced production and secretion of OPN in the liver;

- Defective OPN caused by hepatic CREBZF deficiency improves NASH by repressing fibrogenic genes in hepatic stellate cells;

- Inhibition of miR-6964-3p by CREBZF mediates the production and secretion of OPN in hepatocytes;

- Hyperactivation of CREBZF in the liver exacerbates fibrosis in NASH; 


\section{Introduction}

Nonalcoholic fatty liver disease (NAFLD) is the most common chronic liver disease around the world $^{1-3}$. NAFLD ranges from simple hepatic steatosis to more severe nonalcoholic steatohepatitis (NASH), which may progress to cirrhosis and ultimately to hepatocellular carcinoma (HCC $)^{4,5}$. NASH is characterized by the presence of persistent liver injury, chronic inflammation, and dysfunctional liver fibrosis ${ }^{6}$. Progressive fibrosis is the hallmark of NASH disease progression and liver fibrosis is the leading determinant of long-term mortality in NASH patients ${ }^{7-9}$. It is generally believed that intrahepatic crosstalk plays a crucial role in the progression of $\mathrm{NASH}^{1},{ }^{10}$. However, the exact mechanism of intrahepatic crosstalk on NASH progression, especially on liver fibrosis, remains largely unknown ${ }^{1,10}$.

Cellular networks play a key role in the development of NASH fibrosis. The secretion of profibrogenic mediators from stressed or injured hepatocytes or immune cells, including macrophages, T cells and NKT cells ${ }^{11-13}$ trigger the hepatic stellate cells (HSCs) fibrogenic activity ${ }^{14-16}$. Notably, the direct interaction of hepatocytes with HSCs is one of the most important drivers of fibrosis in $\mathrm{NASH}^{14,17}$. The damaged or dead hepatocytes release the profibrogenic mediators including Taz signaling-regulated Indian hedgehog $(\mathrm{IHH})$ ligand $^{18}$, profibrogenic damage associated molecular patterns (DAMPs) ${ }^{15,19}$ or apoptotic bodies ${ }^{20}$ that contribute directly to HSCs activation. Interestingly, the extracellular matrix protein osteopontin (OPN) is recently identified as a hepatocyte-derived profibrogenic factor in response to Notch-Sox9 signaling activation ${ }^{21}$. Moreover, OPN is also induced by the hedgehog signaling pathway in cholangiocytes or myofiblastic HSCs, leading to liver fibrogenesis via a paracrine or autocrine dependent manner ${ }^{22}$. Mechanistically, OPN induces the activation of HSCs and the production of collagen type-I through integrin $\alpha v \beta 3$ engagement and leads to liver fibrosis ${ }^{19,23,24}$. Although it is known that production and secretion of OPN is induced in hepatocytes in $\mathrm{NASH}^{21,25}$, the mechanism of OPN in mediating the crosstalk beween hepatocytes and HSCs during fibrosis development in NASH remains largerly unknown.

CREB/ATF bZIP transcription factor (CREBZF) is rapidly gaining interest as a key regulator in hepatic metabolism and cell growth ${ }^{26,27}$. CREBZF normally functions as a transcriptional inhibitor to regulate gene expression ${ }^{27,}{ }^{28}$. During refeeding, CREBZF is induced by insulin to inhibit transcription 
levels of Insig-2a in the hepatocyte, leading to the activation of SREBP-1 and fatty acid synthesis ${ }^{29}$. The expression levels of CREBZF are increased in livers of diet-induced obese, genetically obese mice and NAFLD patients ${ }^{29}$. Hepatocyte CREBZF knockout mice are prevented from diet-induced hepatic steatosis. CREBZF hyperactivation may contribute to sustained lipogenesis and NAFLD under selective insulin resistance conditions ${ }^{27}, 29$. Moreover, CREBZF attenuates hepatocyte proliferation and liver regeneration in mice after hepatectomy or carbon tetrachloride treatment by inhibiting the dimerization and transcriptional activity of STAT3 ${ }^{30}$. However, the role of CREBZF in the regulation of NASH progression is unknown.

Recently, we identified hormonal regulation of hepatic steatosis, liver fibrosis and NASH progression in diet-induced obese rodents and nonhuman primates ${ }^{31-33}$. Here, we provide insights into the mechanisms by which a novel intrahepatic crosstalk in the regulation of liver fibrosis and NASH pathogenesis. Gain-of-function and loss-of-function approaches demonstrate the essential role of CREBZF in the regulation of liver fibrosis in NASH. The in vivo and in vitro studies demonstrate that (1) Hepatic CREBZF is required for NASH diet-induced production and secretion of OPN in the liver; (2) Defective OPN caused by hepatic CREBZF deficiency improves NASH by repressing fibrogenic genes in hepatic stellate cells; (3) Inhibition of miR-6964-3p by CREBZF mediates the production and secretion of OPN in hepatocytes; (4) Hyperactivation of CREBZF in mouse livers exacerbates fibrosis in NASH.

\section{Materials and Methods}

Animal model and diets. Liver-specific CREBZF knockout (CREBZF LKO) mice were generated by crossing the floxed CREBZF mice containing the deleted open reading frame of CREBZF (Cyagen Biosciences, China) with albumin-Cre recombinase transgenic mice (Model Animal Research Center of Nanjing University, China), the wild-type (WT) littermates (albumin-Cre negative, CREBZF flox/flox) were used as the control. Male C57BL/6 mice at 8 weeks of age were purchased from Shanghai Laboratory Animal Co. Ltd, China. Mice were fed on the Amylin liver NASH model (AMLN) diet (40\% fat, $22 \%$ fructose and $2 \%$ cholesterol, D09100301, Research Diets) for 20 weeks or methionine and choline deficient (MCD) $\operatorname{diet}\left(\right.$ A02082002B, Research Diets) for 4 weeks ${ }^{1,33,34}$. All mice were housed under a 12:12-h light/dark 
cycle at controlled temperature. All animal experimental protocols were approved by Institutional Animal Care and Use Committee at the Shanghai Institute of Nutrition and Health, Chinese Academy of Sciences, Shanghai, China.

Primary mouse hepatocyte isolation and culture. Primary mouse hepatocytes were isolated using a method described previously $29,30,33$. Briefly, mice were anesthetized with sodium pentobarbital (30 mg/kg intraperitoneally), and the portal vein was cannulated under aseptic conditions. The liver was perfused with ethylene glycol-bis (2-aminoethylether)-N,N,N',N'-tetraacetic acid (EGTA) solution (5.4 mmol// KC1, $0.44 \mathrm{mmol} / / \mathrm{KH}_{2} \mathrm{PO}_{4}, 140 \mathrm{mmol} / / \mathrm{NaCl}, 0.34 \mathrm{mmol} / \mathrm{l} \mathrm{Na} 2 \mathrm{HPO} 4,0.5 \mathrm{mmol} / \mathrm{l} \mathrm{EGTA}, 25 \mathrm{mmol} / \mathrm{l}$ Tricine, $\mathrm{pH}$ 7.2) and Hank's Balanced Salt Solution (HBSS) containing $0.075 \%$ collagenase type I (Sigma-Aldrich), $10 \mathrm{mg} / \mathrm{ml}$ DNase I (Sigma-Aldrich), 200 units $/ \mathrm{ml}$ penicillin, and $200 \mu \mathrm{g} / \mathrm{ml}$ streptomycin, and then digested with $0.025 \%$ collagenase solution for the mouse liver. The isolated mouse hepatocytes were then cultured at $80 \%-90 \%$ confluence in DMEM medium containing 10\% FBS in rat-tail collagen type I-coated 6-well plates (BD Biosciences) overnight.

Generation of adeno-associated viruses (AAV). To construct AAV-TBG-OPN and AAV-TBG-CREBZF, the cDNA encoding GFP in AAV-TBG-GFP vector was replaced with a fragment encoding cDNA sequence of OPN or CREBZF. Three types of DNA vectors are used to package the AAV virus, AAV shuttle vector that contain expressing cassette (OPN, CREBZF or GFP); Delta F6 (DF6) helper plasmid that supplies E2a, E4, VARNA; AAV2/8 RC plasmid (Rev Cap) for specific AAV serotype. The ratio of the above three vectors is 1:1:1. Following transient transfection with PEI and virus package in HEK293T cells, AAV vectors were purified using discontinuous iodixanol gradient centrifugation as previously described with minor modifications ${ }^{35}$. The purified AAV vectors were dissolved and stored in $1 \mathrm{xPBS}$ containing $10^{-4} \mathrm{~F} 188$ and $5 \%$ glycerol, and tittered using a previously described real-time PCR procedure ${ }^{36}$.

Statistical analysis. Data are expressed as mean \pm SEM. Statistical significance was evaluated using the unpaired two-tailed Student's $\mathrm{t}$ test for normally distributed data, the Mann-Whitney rank sum test for non- 
normally distributed data, and one-way ANOVA analysis for more than two groups. Differences were considered significant at a $P$ value $<0.05$.

\section{Results}

Hepatic CRBEZF deficiency protects mice from AMLN diet-induced liver fibrosis in nonalcoholic steatohepatitis

To investigate the role of CREBZF on nonalcoholic steatohepatitis (NASH), a diet-induced model of $\mathrm{NASH}$ was generated in mice fed with the Amylin liver NASH model (AMLN) diet composed of high fat, fructose and cholesterol, which is widely used as a preclinical model for developing human NASH features, including steatosis, inflammation and pericellular fibrosis ${ }^{1,34}$. As shown in Fig. 1A-E, compared with WT mice, CREBZF LKO mice showed marked reduction of most histologic features of NASH, including inflammatory cell infiltration and hepatocytes ballooning. Importantly, CREBZF LKO mice developed less liver fibrosis than WT mice fed with AMLN diet as determined by Sirius Red staining. Plasma levels of alanine aminotransferase (ALT) and aspartate aminotransferase (AST) were significantly decreased in the CREBZF LKO group, suggesting alleviation of hepatocyte damage. As shown in Fig. S1, plasma cholesterol levels were decreased in CREBZF LKO mice. Notably, no obvious changes in plasma triglyceride levels, body weights, body compositions and expression levels of lipogenic genes were observed. These results indicate that CREBZF deficiency in hepatocytes attenuates AMLN diet-induced fibrosis and liver injury in mice.

To understand the effects of CREBZF deficiency on liver transcriptome, a genome-wide transcriptomic analysis by RNA sequencing (RNA-seq) of the liver in mice fed with AMLN diet was performed. As shown in Fig. 1F-H, the analysis identified a total of 1931 differentially expressed genes between CREBZF LKO and WT mice. Compared with WT mice, the genes involved in extracellular matrix (ECM), collagen and cytokine-related pathways were mainly down-regulated in CREBZF LKO mice as analyzed by Kyoto Encyclopedia of Genes and genomes (KEGG) and Gene Ontology (GO) enrichment analysis. Gene Set Enrichment Analysis (GSEA) revealed that CREBZF deficiency is negatively 
correlated with the expression of genes involved in ECM production, chemotaxis and wound healing (Fig. 11 and Fig. S2A).

Given that the liver non-parenchymal cells (NPCs), involved in the regulation of ECM remodeling, immune response and cell adhesion, are critical for NASH pathogenesis, single-cell RNA sequencing (scRNA-seq) was performed. The results were visualized by T-distributed Stochastic Neighbor Embedding (t-SNE) showing seven major clusters corresponding to T cells, B cells, macrophages, NK cells, mast eosinophils, neutrophils and dendritic cells (Fig. 1J). The transcriptomic signature of macrophages was further divided into 5 clusters (Fig. S2B). Interestingly, CREBZF LKO mice had much lower ratio of cluster 3 macrophage, most of which express genes involved in the proinflmmatory pathways including toll-like receptor (TLR), tumor necrosis factor (TNF) and NF-kB signaling pathways (Fig. 1K). Taken together, these data show that hepatic CREBZF deficiency protects against diet-induced collagen deposition, fibrosis and NASH progression, and that the reduction of the pro-inflammatory and pro-fibrotic microenvironment in livers of CREBZF LKO mice may play roles in the attenuation of NASH progression.

\section{Loss of CREBZF in hepatocytes suppresses AMLN diet-induced production and secretion of hepatic OPN}

To identify the underlying mechanism that mediates the intrahepatic crosstalk, analysis of differentially regulated genes enriched in the regulation of cell communication were performed using the RNA-seq data of mouse livers (Fig. 2A). Interestingly, OPN, a secreted extracellular matrix protein involved in the pathogenesis of fibrosis and NASH progression, was significantly reduced in livers of CREBZF LKO mice compared with those of WT mice fed with AMLN diet (Fig. 2B). As shown in Fig. 2CE and S3, consistent with the previous studies showing increased expression of hepatic and circulating

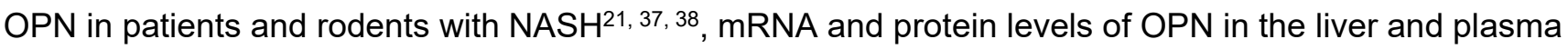
were significantly elevated in mice fed with AMLN diet. Strikingly, compared with WT mice, CREBZF deficiency caused a robust reduction of OPN as well as its target fibrogenic genes, including TGF $\beta$, CTGF, Acta2, Col1a1, Col3a1, Col4a1 and Timp1 (Fig. 2F). Consistently, mRNA levels of proinflammatory 
genes were reduced in livers of CREBZF LKO mice fed with AMLN diet, including TNFa, IL-1 $\beta$, CCL2 and CD68 (Fig. 2G). To further investigate the roles of CREBZF in NASH, a MCD diet-induced mouse model was used. As shown in Fig. S4, consistent with the results in mice fed with AMLN diet, CREBZF deficiency in hepatocytes caused a potent reduction of hepatic fibrosis and inflammation, and plasma ALT and AST levels in mice fed with MCD diet. Moreover, the mRNA levels of OPN and its target fibrogenic genes, and proinflammatory genes were significantly reduced in livers of CREBZF LKO mice. Taken together, these results demonstrate that hepatic CREBZF is necessary for AMLN and MCD dietinduced gene transcription, protein production and secretion of OPN.

\section{Expression levels of fibrogenic genes in HSCs are activated by CREBZF-induced OPN in} hepatocytes

Next, the mechanisms of hepatocyte CREBZF on stimulating fibrogenesis were rigorously assessed in multiple cells, including hepatocytes, HSCs and macrophages. As shown in Fig. 3A-C, CREBZF deficiency caused a markedly reduced production and secretion of OPN in hepatocytes of mice fed with AMLN diet or in hepatocytes exposed to palmitic acid (PA) and TNFa, which has been used to mimic NASH-induced liver injury ${ }^{39}$. Given that mRNA levels of fibrosis-related genes were up-regulated by treatment with recombinant OPN (rOPN) in HSC-T6 cells (Fig. 3D), we hypothesize that CREBZF might increase OPN expression in hepatocytes to stimulate activation of HSCs and induction of fibrogenic gene expression. To test this hypothesis, cell co-culture assays were performed. As shown in Fig. 3F, the conditioned medium (CM) from hepatocytes exposed to PA and TNFa markedly increased the expression of fibrogenic genes in HSC-T6 cells, including CTGF, Acta2, Col4a1 and Timp1, whereas these effects were abrogated by CREBZF deficiency in hepatocytes. Strikingly, reconstitution of rOPN in the conditioned medium isolated from CREBZF-/- hepatocytes exposed to PA and TNFa restored the expression of fibrogenic gene expression in HSC-T6 cells, suggesting that hepatocyte-derived OPN may mediate the effects of CREBZF on fibrogenesis in HSC-T6 cells.

The effects of hepatic CREBZF deficiency on activation of macrophages were also assessed in RAW 264.7 cells. Although expression levels of proinflammatory genes including TNFa, IL-1 1 and CCL2 
were increased in RAW 264.7 cells treated with CM from hepatocytes exposed to PA and TNFa, no obvious changes were observed whether treated with CM from CREBZF+/+ or that from CREBZF-/hepatocytes (Fig. 3G). Taken together, these data suggest that hepatocyte-derived OPN likely couples fibrogenic signaling from the hepatocytes to the activation of HSCs, instead of macrophages.

\section{Hepatic OPN restoration ablates the protection of liver fibrosis in CREBZF LKO mice}

To directly assess whether suppression of OPN is responsible for improvement of liver fibrosis and NASH progression in CREBZF LKO mice, CREBZF LKO and WT mice were treated with AAV-encoding OPN or GFP via tail vein injection for 1 week, followed by MCD diet for 4 weeks. As shown in Fig. 4A-E, compared with CREBZF LKO mice injected with AAV-GFP, hepatic overexpression of OPN caused a significant induction of liver fibrosis which is determined by Sirius Red staining and fibrosis stage evaluation. Plasma levels of ALT and AST were significantly increased in AAV-OPN-injected mice, suggesting aggravation of hepatocyte damage. Consistently, hepatic overexpression of OPN caused a robust induction of its target fibrogenic genes in CREBZF LKO mice fed with MCD diet, including Acta2, Col1a1, Col4a1 and Timp1 (Fig. 4F, G). Therefore, these results further demonstrate that hepatic CREBZF deficiency ameliorates diet-induced liver fibrosis and NASH progression largely through hepatic impairment of OPN.

\section{CREBZF inhibits miR-6964-3p to promote the production and secretion of OPN in hepatocytes}

Given that the association between CREBZF and Sox9 or Hes1, the key transcription factors that promote transcription levels of OPN in the liver or osteoblasts ${ }^{21,40,41}$, was not observed (data not shown), it is unlikely that CREBZF regulates OPN directly. To investigate the direct target of CREBZF in hepatocytes, analysis by microRNA sequencing (miRNA-seq) of the liver in mice fed with AMLN diet was performed. MicroRNA-6964-3p (miR-6964-3p), a novel microRNA that likely binds the 3'-untranslated region (UTR) of OPN as predicted by Targetscan database ${ }^{42}$, was significantly increased in livers of CREBZF LKO mice compared with those of WT mice fed with AMLN diet (Fig. 5A). Given that CREBZF normally acts as an inhibitor to repress target gene transcription ${ }^{26,29,30}$, we therefore hypothesize that 
CREBZF inhibits the transcription of miR-6964-3p to increase the production and secretion of OPN in hepatocytes.

To test this hypothesis, effects of mimics of miR-6964-3p together with other microRNAs identified by miRNA-seq analysis, including miR-6383, let-7c-1-3p, miR-494-3p, on the expression of OPN were verified in AML12 hepatocytes by real-time PCR. Consistent with the effects of miR-186-5p, which is known to inhibit OPN in chondrocytes cells ${ }^{43}$, treatment with miR-6964-3p mimic caused a potent reduction of OPN in AML12 hepatocytes. Importantly, treatment with miR-6964-3p mimic also caused a significant reduction of the luciferase activity of OPN-WT 3' UTR, whereas these effects were largely ablated in cells transfected with the mutant reporter OPN-Mut 3' UTR (Fig. 5B). The inhibitory effects of miR-6964-3p on OPN were further verified in mouse primary hepatocytes by miR-6964-3p mimic or inhibitor (Fig. 5C, D). These results indicate that OPN is likely a downstream target of miR-6964-3p.

In contrast to CREBZF LKO mice, CREBZF overexpression caused a significant reduction of miR6964-3p, leading to an induction of OPN in AML12 hepatocytes (Fig. 5E, F). To further elucidate the causal effects of CREBZF on inhibiting miR-6964-3p and augmentation of OPN, primary hepatocytes were treated with PA and TNFa, mimicking NASH conditions in vivo ${ }^{39,44}$. As shown in Fig. 5G-H, consistent with the results in CREBZF LKO mice fed with AMLN diet, CREBZF deficiency caused a significant induction of miR-6964-3p in mouse primary hepatocytes, whereas expression levels of OPN were significantly repressed. Strikingly, reduced expression levels of OPN in CREBZF deficient hepatocytes exposed to PA and TNFa were restored by treatment with miR-6964-3p inhibitor, suggesting that miR-6964-3p inhibition mediates the stimulatory effects of CREBZF on OPN. Consistently, compared with WT mice, expression levels of miR-6964-3p were increased in livers of CREBZF LKO mice fed with AMLN or MCD diet (Fig. 5I). Taken together, these results indicate that inhibition of miR-6964-3p mediates CREBZF-induced production and secretion of OPN in hepatocytes, and may play important roles in liver fibrosis and NASH progression.

Hepatic inflammation, liver injury and fibrosis are exacerbated by adeno-associated
overexpression of CREBZF in mice fed with MCD diet.


To investigate the gain-of-function effects of CREBZF on liver fibrosis and NASH progression, AAVmediated gene delivery was performed in mice fed with MCD diet (Fig. 6A). As shown in Fig. 6B-E, compared with control GFP mice, the livers of AAV-CREBZF-treated mice had more collagen deposition, infiltration of inflammatory cells and elevated levels of plasma ALT and AST, suggesting aggravation of hepatic inflammation, liver injury and fibrosis.

In contrast to the phenotypes in CREBZF LKO mice fed with AMLN or MCD diet, overexpression of CREBZF in the liver caused a significant reduction of miR-6964-3p and augmentation of OPN, leading to an induction of fibrogenic genes, including TGF $\beta$, Acta2, Col1 1 1, Col3a1, Col4a1 and Timp1 (Fig. 6FH). Consistently, mRNA levels of proinflammatory genes were also increased in livers of CREBZF-treated mice, including TNFa, IL-1 $\beta$ and CCL2 (Fig. 6I). Notably, overexpression of CREBZF in the liver were verified by immunoblots (Fig. 6J). Importantly, compared with chow-fed mice, protein levels of CREBZF in the liver were significantly higher in AMLN or MCD diet-induced mice, suggesting a positive correlation of CREBZF activation in the development of NASH phenotypes (Fig. $6 \mathrm{~K}, \mathrm{~L}$ ). Taken together, these results suggest that CREBZF may be linked to the pathogenesis of hepatic inflammation, liver injury and fibrosis.

\section{Discussion}

The current study reveals an intrahepatic crosstalk between hepatocytes and HSCs in the regulation of liver fibrosis and NASH progression. CREBZF functions as a bona fide upstream regulator of key fibrogenic extracellular matrix protein OPN in hepatocytes. The increased production and secretion of OPN by CREBZF leads to activation of HSCs and progression of liver fibrosis in a paracrine manner. Inhibition of a novel microRNA miR-6964-3p mediates CREBZF-stimulated OPN in hepatocytes. Hepatocyte CREBZF-OPN axis may represent a molecular mechanism by which intrahepatic signals from injured hepatocytes are transduced to HSCs and then regulate liver fibrosis in nonalcoholic steatohepatitis (Fig. 7).

\section{CREBZF serves as a novel regulator of OPN}


This study characterizes CREBZF as a novel regulator of OPN in hepatocytes. CREBZF gain- and loss-of-function approaches demonstrate stimulatory effects of CREBZF on hepatic OPN production and secretion in mouse livers, primary hepatocytes and hepatocyte cell lines. CREBZF deficiency in hepatocyte significantly decreased the expression levels of OPN in livers of mice fed with AMLN and MCD diets. Furthermore, in vitro cell treatments showed the secretion of OPN from primary hepatocytes and hepatic CREBZF deficiency decreased OPN expression in hepatocytes isolated from AMLN diet-fed mice or treated with PA and TNFa. In vivo and in vitro assays demonstrated that CREBZF stimulates the expression and secretion of OPN in hepatocytes.

Our results demonstrated a microRNA-dependent regulation of CREBZF on OPN expression. Mechanistically, CREBZF inhibits the expression of miR-6964-3p, and the reduction of miR-6964-3p causes a potent induction of OPN likely via the binding of miR-6964-3p to the 3' UTR of OPN. Therefore, CREBZF functions as a positive regulator of OPN indirectly through inhibiting miR-6964-3p, which is consistent with the important roles of microRNAs in the regulation of liver functions ${ }^{45}$. These findings are consistent with the actions of CREBZF as an inhibitor for Insig-2a to promote sustained lipogenesis in response to refeeding or during diet-induced obesity in mice ${ }^{29}$, or for STAT3 to repress hepatocyte proliferation and liver regeneration after hepatectomy in mice ${ }^{30}$. Although OPN is induced by Notch target Sox $9^{21}$, the transcritptional regulation of OPN remains largely unknown. The current study characterizes CREBZF-miR-6964-3p axis as a novel mechanism in regulating OPN production and secretion in hepatocytes. Together, these results demonstrate that CREBZF acts as a positive regulator of OPN, and support a finely tuned cellular mechanism to increase OPN activity in stressed or injured hepatocytes.

\section{The crosstalk between hepatocytes and HSCs is mediated by CREBZF-OPN axis in NASH}

This study utilizes in vivo and in vitro approaches to demonstrate previously unrecognized mechanisms of hepatic CREBZF in regulating nonalcoholic steatohepatitis. Although previous studies suggested that hepatic CREBZF increases lipogenesis to promote hepatic steatosis ${ }^{27,29}$, none has linked hepatocyte CREBZF to fibrosis in the setting of NASH. First, CREBZF deficiency in hepatocytes alleviates hepatic inflammation, liver injury and fibrosis in AMLN or MCD diet-fed mice. These results are consistent 
with recent findings showing that CREBZF is essential for hepatocyte proliferation and liver regeneration, and that CREBZF deficiency in hepatocytes results in improvement of inflammation and liver injury after hepatectomy or $\mathrm{CCl}_{4}$ treatment ${ }^{30}$. Second, the present study utilizes cell-based assays, including mouse primary hepatocytes, AML12 hepatocytes and HSC-T6, to rigorously demonstrate the crosstalk between hepatocytes and HSCs. These data demonstrate that hepatocyte CREBZF-OPN axis controls liver fibrosis and NASH progression through direct activation of HSCs. Importantly, the protection of liver fibrosis in CREBZF LKO mice fed with MCD diet is ablated by hepatic OPN restoration, strongly indicating that CREBZF-OPN axis plays essential roles in mediating intrahepatic crosstalk between hepatocytes and HSCs in the progression of NASH. Together, we utilized classical diet-indued NASH rodent models together with cell co-culture assays to demonstrate that the crosstalk between hepatocytes and HSCs is critical for hepatic inflammation, liver injury and hepatic fibrogenesis, and CREBZF-OPN axis plays important roles in the pathogenesis of NASH.

\section{Therapeutic implication of CREBZF in NASH}

The current study demonstrates that CREBZF inhibits miR-6964-3p to promote OPN production and secretion in hepatocytes, leading to activation of HSCs and liver fibrosis, and characterizes CREBZF as a novel upstream regulator of OPN pathway. Hepatocyte-specific deletion of CREBZF protects mice from AMLN or MCD diet-induced liver fibrosis in nonalcoholic steatohepatitis. In contrast, AAV-mediated liver-specific overexpression of CREBZF aggravates the pathogenesis of $\mathrm{NASH}$, including more inflammatory cell infiltrations and collagen depositions in livers of mice fed with MCD diet. NASH is an extreme outcome of NAFLD, these results demonstrate that hyperactivation of CREBZF in the liver may be a cause of the progressive lesion of NASH, which is consistent with the detrimental role of CREBZF in liver injury after hepatectomy ${ }^{30}$. Moreover, consistent with the increased expression of CREBZF in livers of diet-induced obese and genetically obese mice, and NAFLD patients ${ }^{29}$, expression levels of CREBZF are significantly induced in the NASH livers of mice fed with AMLN or MCD diet. However, comparing with WT mice, CREBZF LKO mice did not show significant changes in hepatic steatosis in AMLN diet induced NASH model. In MCD diet-induced NASH mice that represents weight loss and insulin 
sensitivity, hepatic CREBZF deficiency decreases collagen deposition in the liver. These results indicate that the fibrosis stimulating effects of CREBZF are unlikely to be mediated through the aggravation of lipid metabolism. Therefore, we propose that in response to different cellular triggers, CREBZF may represent an essential regulator in the transition from simple steatosis to $\mathrm{NASH}$. And pharmacological targeting of CREBZF could represent an appealing strategy for the treatment of dysregulation of lipid metabolism and collagen deposition during the progression of NAFLD. NASH is already become the second leading etiology of liver disease among adults awaiting liver transplantation in the United States ${ }^{46}$. Given that hepatic fibrosis is the leading determinant of long-term mortality in patients with NASH ${ }^{7-9}$ and organ availability for transplantation is limited, new pharmaceutical targets to address $\mathrm{NASH}$-associated fibrosis are a large unmet need for an increasingly NASH population. Therefore, hepatic inhibition of CREBZF may have the potential for the improvement of liver fibrosis and NASH.

In summary, the current study reveals a novel mechanism of hepatocyte CREBZF-OPN axis in the control of liver fibrosis in nonalcoholic steatohepatitis. The inhibition of miR-6964-3p by CREBZF leads to hepatocyte-derived OPN production and secretion, resulting in increased profibrotic activity of HSCs and liver fibrosis in NASH. Pharmacological and genetic approaches for the inhibition of CREBZF activity may provide a potential strategy for the treatment of liver fibrosis and NASH.

\section{Acknowledgments}

This work was supported by grants from National Key R\&D Program of China (2019YFA0802502), National Natural Science Foundation of China (81925008), Key Laboratory of Wuliangye-flavor Liquor Solid-state Fermentation of China National Light Industry (2019JJ005), Shanghai Science and Technology Commission (19140903300) to Y.L. This work was also supported by grants from China Postdoctoral Science Foundation (2021M693273) to A.C. We are grateful to Dr. Zhuoxian Meng (Zhejiang University) for providing vectors encoding adeno-associated viruses, and Zehua Zhao (Shanghai Jiaotong University), Jinyun Bai (Fudan University), Yamei Han, Feifei Zhang, Chao Sun, Jing Gao (SINH) for technical assistance. We would also like to thank Zhonghui Weng and Yifan Bu from Institutional Center for Shared Technologies and Facilities of SINH, CAS for technical assistance. 


\section{Figure Legends}

Fig.1. Hepatocyte-specific deletion of CREBZF protects mice from AMLN diet-induced liver fibrosis in nonalcoholic steatohepatitis. Male liver-specific CREBZF knockout (CREBZF LKO) mice and their wild-type littermates (WT) at 8 weeks old were fed a chow diet or AMLN diet for 20 weeks. (A) Representative H\&E and Sirius Red staining of liver sections (scale bars: $50 \mu \mathrm{m}$ ). (B) Reduction of plasma ALT and AST levels in CREBZF LKO mice fed with AMLN diet. $n=7-8,{ }^{*} p<0.05$, versus WT and chow; $\# p<0.05$, versus WT and AMLN. (C) Quantification of the Sirius Red positive area in the liver sections. $n=6-8,{ }^{*} p<0.05$, versus WT and chow; \#p<0.05, versus WT and AMLN. (D, E) Pathological scores of steatosis, lobular inflammation and hepatocellular ballooning and NASH CRN scores (D) and fibrosis stage (E) in CREBZF LKO and WT mice fed with AMLN diet for 20 weeks. $n=9-11,{ }^{*} p<0.05$, versus WT. (F-I) Clustering analysis of up-regulated or down-regulated genes (F), Kyoto Encyclopedia of Genes and Genomes (KEGG) enrichment analysis (G), Gene Ontology (GO) enrichment analysis (H), and Gene Set Enrichment Analysis (GSEA) (I) of the liver RNA-seq data from CREBZF LKO and WT mice fed with AMLN diet for 20 weeks $(n=4)$. (J) t-SNE visualization of liver cell clusters based on 8697 single-cell transcriptomes of nonparenchymal cells isolated from livers of 6 pairs of CREBZF LKO and WT mice fed with AMLN diet for 20 weeks. (K) Clusters of annotated macrophages displayed in the single-cell RNA sequencing data. (L) Expression levels of CREBZF in livers of CREBZF LKO and WT mice were measured by real-time PCR. $n=8,{ }^{*} p<0.05$, versus WT.

Fig.2. Expression levels of OPN are down-regulated in livers of CREBZF LKO mice fed with AMLN diet. Male CREBZF LKO and WT mice at 8 weeks old were fed a chow diet or AMLN diet for 20 weeks. (A) Heatmap of the gene expression of the 'regulation of cell communication' term (GO: 0010646) $(n=4)$.

(B) Scatter diagram of the significant differentially expressed genes in livers of CREBZF LKO and WT mice fed with 20 weeks AMLN diet $(n=4)$. (C-E) Hepatic expression of OPN and fibrosis related gene Col1a1 are increased in mice fed with AMLN diet, and the induction is eliminated in CREBZF LKO mice. (C) Transcription levels of OPN were measured by real-time PCR. $n=7-8,{ }^{*} p<0.05$, versus WT and chow; $\# p<0.05$, versus WT and AMLN. Immunoblot analysis of OPN and Col1a1 protein were performed (D), 
and the band intensity was quantified by densitometry (E). $n=3,{ }^{*} p<0.05$, versus WT and chow; $\# p<0.05$, versus WT and AMLN. $(F, G)$ Expression levels of hepatic fibrosis related genes $(F)$ and proinflammatory genes $(G)$ are decreased in CREBZF LKO mice. mRNA levels were measured by real-time PCR. $n=6-8$, ${ }^{*} \mathrm{p}<0.05$, versus WT.

Fig.3. CREBZF stimulates the production and secretion of OPN in hepatocytes to activate fibrogenic gene expression in HSCs. (A) AMLN diet-induced expression of OPN is ablated in CREBZF/- hepatocytes. CREBZF+/+ and CREBZF-/-mouse primary hepatocytes were isolated from mice fed with chow diet and AMLN diet for 20 weeks. mRNA levels of OPN were measured by real-time PCR. $n=3-4$, ${ }^{*} p<0.05$, versus CREBZF+/+ and chow; $\# p<0.05$, versus $C R E B Z F+/+$ and $A M L N .(B, C) C R E B Z F$ deficiency attenuates palmitic acid (PA) and TNFa (PA/TNFa) induced production and secretion of OPN in hepatocytes. CREBZF+/+ and CREBZF-/- mouse primary hepatocytes were treated with $250 \mu \mathrm{M}$ PA and $10 \mathrm{ng} / \mathrm{ml}$ TNFa for 24 hours. mRNA levels of OPN were assessed by real-time PCR (B), the concentrations of OPN in media were assayed by ELISA (C). $n=4,{ }^{*} p<0.05$, versus CREBZF $+/+$ and Vehicle; \#p<0.05, versus CREBZF+/+ and PA and TNFa. (D) Recombinant OPN (rOPN) promotes expression of fibrogenesis-related genes in HSCs. HSC-T6 cells were treated without or with $10 \mathrm{ng} / \mathrm{ml}$, $30 \mathrm{ng} / \mathrm{ml}$ and $100 \mathrm{ng} / \mathrm{ml}$ rOPN for 24 hours, mRNA levels of profibrotic genes ACTA2, Col1a1and CTGF were measured by real-time PCR. $n=4,{ }^{*} p<0.05$, versus PBS. (E, F) CREBZF deficient hepatocyteconditioned medium decreases fibrogenic genes expression in HSCs, and the reduction is reversed by rOPN treatment. CREBZF+/+ and CREBZF-/- mouse primary hepatocytes were treated with $250 \mu \mathrm{M} P A$ and $10 \mathrm{ng} / \mathrm{ml} \mathrm{TNFa}$ for 24 hours, then were incubated in fresh serum free medium, and the conditioned medium were collected after 24 hours. The conditioned medium without or with $10 \mathrm{ng} / \mathrm{ml} \mathrm{rOPN}$ were then transferred to HSC-T6 cells for 24 hours. (E) The schematic diagram of the preparation of conditioned medium and cell treatment. (F) mRNA levels of fibrosis-related genes CTGF, Acta2, Col4a1 and Timp1 in HSC-T6 cells were assessed. $n=5,{ }^{*} p<0.05$, versus CREBZF+/+ and Vehicle; $\# p<0.05$, versus CREBZF+/+ and PA and TNFa; $\$ p<0.05$, versus CREBZF-/- and PA and TNFa. (G) CREBZF deficient hepatocyte conditioned medium causes no obvious effects on the expression of proinflammatory genes 
in the macrophage. mRNA levels of TNFa, IL-1 $\beta$ and CCL2 were measured in RAW 264.7 cells incubated with primary hepatocyte conditioned medium. $n=4,{ }^{*} p<0.05$, versus CREBZF $+/+$ and Vehicle; $\# p<0.05$, versus CREBZF+/+ and PA and TNFa.

Fig.4. The protection of liver fibrosis in CREBZF LKO mice fed with MCD diet is ablated by hepatic OPN restoration. Male CREBZF LKO and WT mice at 10 weeks old were treated with AAV-encoding OPN or GFP via tail vein injection for 1 week, followed by MCD diet for 4 weeks. (A) The schematic diagram of the animal experiment. (B) Representative H\&E and Sirius Red staining of liver sections (scale bar, $50 \mu \mathrm{m})$. (C) The alleviation of plasma ALT and AST levels in CREBZF LKO mice by AAV-OPN injection. $n=8-9,{ }^{*} p<0.05$, versus WT and AAV-GFP; $\# p<0.05$, versus LKO and AAV-GFP. (D, E) AAVOPN treatment promotes hepatic fibrosis in CREBZF LKO mice. The quantification of the Sirius Red positive area (D) and fibrosis stage of the liver sections (E) were assessed. $n=9-10,{ }^{*} p<0.05$, versus WT and AAV-GFP; \#p<0.05, versus LKO and AAV-GFP. $(F, G)$ Hepatic overexpression of OPN increases the expression of fibrogenic genes in the liver of CREBZF LKO mice. (F) mRNA levels of fibrosis-related genes were measured by real-time PCR. $n=6-8$, * $p<0.05$, versus WT and AAV-GFP; $\# p<0.05$, versus LKO and AAV-GFP. (G) Immunoblot analysis of OPN and Col1a1 were performed, and the band intensity was quantified. $n=3,{ }^{*} p<0.05$, versus WT and AAV-GFP; $\# p<0.05$, versus LKO and AAV-GFP.

Fig.5. Inhibition of miR-6964-3p mediates CREBZF-stimulated production and secretion of OPN in hepatocytes. (A) Heatmap of hepatic microRNAs in livers of CREBZF LKO and WT mice fed with AMLN diet for 20 weeks $(n=4)$. (B) miR-6964-3p targets 3' UTR of the OPN mRNA and decreases mRNA levels of OPN. Graphic representation of the miR-6964-3p binding motif within the 3'UTR of OPN gene. Complementary wild type (WT) and mutated (Mut) seed sequence of miR-6964-3p within the 3'UTR are shown. Mimics of miR-6964-3p and other microRNAs were transfected into AML12 hepatocytes for 48 hours and mRNA levels of OPN were measured by real-time PCR. $n=3,{ }^{*} p<0.05$, versus negative control. For luciferase reporter assay, HEK293T cells were transfected with WT-OPN 3' UTR-Luc or Mut-OPN 3' UTR-Luc together with miR-6964-3p mimic or negative control for 48 hours. Relative luciferase activities were determined. $n=4,{ }^{*} p<0.05$, versus negative control and WT. (C, D) miR-6964-3p acts as a repressor 
of OPN in primary hepatocytes. miR-6964-3p mimic (C) and inhibitor (D) were transfected into primary hepatocytes, mRNA levels of OPN were measured by real-time PCR. $n=4,{ }^{*} p<0.05$, versus negative control mimic or negative control inhibitor. (E, F) CREBZF inhibits the expression of miR-6964-3p and increases mRNA levels of OPN in AML12 hepatocytes. FLAG-tagged CREBZF or pcDNA were transfected in AML12 hepatocytes, mRNA levels of miR-6964-3p (E) and OPN (F) were assessed. $n=4$, ${ }^{*} p<0.05$, versus pcDNA. (G) Expression of miR-6964-3p is increased in CREBZF deficient hepatocytes. CREBZF+/+ and CREBZF-/- mouse primary hepatocytes were treated without or with $250 \mu \mathrm{M}$ PA and 10 $\mathrm{ng} / \mathrm{ml}$ TNFa for 24 hours. Expression levels of miR-6964-3p were measured by real-time PCR. $\mathrm{n}=4$, ${ }^{*} \mathrm{p}<0.05$, versus CREBZF+/+ and Vehicle; $\# p<0.05$, versus CREBZF+/+ and PA and TNFa. $(\mathrm{H})$ Inhibition of miR-6964-3p reverses expression of OPN in CREBZF deficient primary hepatocytes exposed to PA and TNFa. After a 48-hour period of transfection with miR-6964-3p inhibitor or negative control inhibitor, CREBZF+/+ and CREBZF-/- mouse primary hepatocytes were treated without or with PA and TNFa for 24 hours. Real-time PCR analysis was performed. $n=4$, * $p<0.05$, versus CREBZF+/+ and Vehicle; $\# p<0.05$, versus CREBZF+/+ and PA and TNFa; $\$ p<0.05$, versus CREBZF-/- and PA and TNFa. (I) Transcription levels of miR-6964-3p are increased in livers of CREBZF LKO mice fed with AMLN diet for 20 weeks or MCD diet for 4 weeks. $n=9,{ }^{*} p<0.05$, versus WT.

Fig.6. Hepatic overexpression of CREBZF exacerbates inflammation, liver injury and fibrosis in mice fed with MCD diet. Male C57BL/6 mice at 10 weeks old were treated with AAV-encoding CREBZF or GFP through tail vein injection for 1 week, followed by MCD diet for 4 weeks. (A) Schematic representation of the animal experiments. (B) Representative H\&E and Sirius Red staining of liver sections (scale bars: $50 \mu \mathrm{m}$ ). (C) Induction of plasma ALT and AST levels in AAV-CREBZF-treated mice fed with MCD diet for 4 weeks. $n=10$, * $p<0.05$, versus AAV-GFP. (D, E) Hepatic fibrosis is aggravated in the mice treated with AAV-CREBZF. The quantification of the Sirius Red positive area (D) and fibrosis stage of the liver sections (E) were assessed. $n=9-10$, ${ }^{*} p<0.05$, versus AAV-GFP. (F-I) The expression levels of miR-6964-3p (F), OPN (G), fibrosis-related genes $(\mathrm{H})$ and proinflammatory genes $(\mathrm{I})$ in the liver were determined by real-time PCR. $n=8-10,{ }^{*} p<0.05$, versus AAV-GFP. (J) Expression levels of CREBZF 
in livers of mice treated with AAV-CREBZF or AAV-GFP were measured by immunoblots. $(K, L)$ Expression levels of CREBZF are elevated in livers of diet-induced NASH mice. Immunoblot analysis of CREBZF and Col1 $\alpha 1$ and quantification of the band intensity in livers of mice fed with chow diet or AMLN $\operatorname{diet}(\mathrm{K})$ and chow diet or MCD diet $(\mathrm{L})$ are shown. $\mathrm{n}=5-7,{ }^{*} \mathrm{p}<0.05$, versus chow.

Fig.7. The proposed model for hepatic CREBZF in regulating the progression of nonalcoholic steatohepatitis: potential therapeutic implication in NASH-associated hepatic fibrogenesis. In the process of the transition from simple steatosis to NASH, CREBZF in hepatocytes is activated and triggers hepatic stellate cells activation and progressive fibrogenesis in the liver. CREBZF stimulates the expression and secretion of fibrogenic factor OPN in hepatocytes by inhibiting miR-6964-3p.

\section{Reference}

1. Friedman SL, Neuschwander-Tetri BA, Rinella M, Sanyal AJ. Mechanisms of nafld development and therapeutic strategies. Nature medicine. 2018;24:908-922

2. Younossi ZM, Koenig AB, Abdelatif D, Fazel Y, Henry L, Wymer M. Global epidemiology of nonalcoholic fatty liver disease-meta-analytic assessment of prevalence, incidence, and outcomes. Hepatology. 2016;64:73-84

3. Younossi Z, Anstee QM, Marietti M, Hardy T, Henry L, Eslam M, George J, Bugianesi E. Global burden of nafld and nash: Trends, predictions, risk factors and prevention. Nature Reviews Gastroenterology \& Hepatology. 2018;15:11-20

4. Cohen JC, Horton JD, Hobbs HH. Human fatty liver disease: Old questions and new insights. Science. 2011;332:1519-1523

5. Anstee QM, Reeves HL, Kotsiliti E, Govaere O, Heikenwalder M. From nash to hcc: Current concepts and future challenges. Nature reviews. Gastroenterology \& hepatology. 2019;16:411-428

6. Diehl AM, Day C. Cause, pathogenesis, and treatment of nonalcoholic steatohepatitis. The New England journal of medicine. 2017;377:2063-2072

7. Angulo P, Kleiner DE, Dam-Larsen S, Adams LA, Bjornsson ES, Charatcharoenwitthaya P, Mills PR, Keach JC, Lafferty HD, Stahler A, Haflidadottir S, Bendtsen F. Liver fibrosis, but no other histologic features, is associated with long-term outcomes of patients with nonalcoholic fatty liver disease. Gastroenterology. 2015;149:389-397 e310

8. Puche JE, Saiman Y, Friedman SL. Hepatic stellate cells and liver fibrosis. Comprehensive physiology.1473-1492.

9. Angulo P, Machado MV, Diehl AM. Fibrosis in nonalcoholic fatty liver disease: Mechanisms and clinical implications. Seminars in liver disease. 2015;35:132-145

10. Schuster S, Cabrera D, Arrese M, Feldstein AE. Triggering and resolution of inflammation in nash. Nature Reviews Gastroenterology \& Hepatology. 2018;15:349-364

11. Krenkel $O$, Tacke F. Macrophages in nonalcoholic fatty liver disease: A role model of pathogenic immunometabolism. Seminars in liver disease. 2017;37:189-197

12. Luo X, Li H, Ma L, Zhou J, Guo X, Woo SL, Pei Y, Knight LR, Deveau M, Chen Y, Qian X, Xiao X, Li Q, Chen X, Huo Y, McDaniel K, Francis H, Glaser S, Meng F, Alpini G, Wu C. Expression of sting is increased in liver tissues from patients with nafld and promotes macrophage-mediated hepatic inflammation and fibrosis in mice. Gastroenterology. 2018;155:1971-1984 e1974

13. Machado MV, Diehl AM. Pathogenesis of nonalcoholic steatohepatitis. Gastroenterology. 2016;150:1769-1777 
14. Zhu C, Tabas I, Schwabe RF, Pajvani UB. Maladaptive regeneration - the reawakening of developmental pathways in nash and fibrosis. Nature reviews. Gastroenterology \& hepatology. 2020

15. Tsuchida T, Friedman SL. Mechanisms of hepatic stellate cell activation. Nature reviews. Gastroenterology \& hepatology. 2017;14:397-411

16. Koyama Y, Brenner DA. Liver inflammation and fibrosis. The Journal of clinical investigation. 2017;127:55-64

17. Schwabe RF, Tabas I, Pajvani UB. Mechanisms of fibrosis development in nonalcoholic steatohepatitis. Gastroenterology. 2020;158:1913-1928

18. Wang X, Zheng Z, Caviglia JM, Corey KE, Herfel TM, Cai B, Masia R, Chung RT, Lefkowitch JH, Schwabe RF, Tabas I. Hepatocyte taz/wwtr1 promotes inflammation and fibrosis in nonalcoholic steatohepatitis. Cell metabolism. 2016;24:848-862

19. Arriazu E, Ge X, Leung T-M, Magdaleno F, Lopategi A, Lu Y, Kitamura N, Urtasun R, Theise N, Antoine DJ, Nieto N. Signalling via the osteopontin and high mobility group box-1 axis drives the fibrogenic response to liver injury. Gut. 2017;66:1123-1137

20. Zhan S-S, Jiang JX, Wu J, Halsted C, Friedman SL, Zern MA, Torok NJ. Phagocytosis of apoptotic bodies by hepatic stellate cells induces nadph oxidase and is associated with liver fibrosis in vivo. Hepatology. 2006;43:435-443

21. Zhu C, Kim K, Wang X, Bartolome A, Salomao M, Dongiovanni P, Meroni M, Graham MJ, Yates KP, Diehl AM, Schwabe RF, Tabas I, Valenti L, Lavine JE, Pajvani UB. Hepatocyte notch activation induces liver fibrosis in nonalcoholic steatohepatitis. Science translational medicine. 2018;10

22. Syn W-K, Choi SS, Liaskou E, Karaca GF, Agboola KM, Oo YH, Mi Z, Pereira TA, Zdanowicz M, Malladi $P$, Chen $Y$, Moylan $C$, Jung $Y$, Bhattacharya SD, Teaberry V, Omenetti A, Abdelmalek MF, Guy CD, Adams DH, Kuo PC, Michelotti GA, Whitington PF, Diehl AM. Osteopontin is induced by hedgehog pathway activation and promotes fibrosis progression in nonalcoholic steatohepatitis. Hepatology. 2011;53:106-115

23. Xiao X, Gang Y, Gu Y, Zhao L, Chu J, Zhou J, Cai X, Zhang H, Xu L, Nie Y, Wu K, Liu Z, Fan D. Osteopontin contributes to tgf- $\beta 1$ mediated hepatic stellate cell activation. Digestive Diseases and Sciences. 2012;57:2883-2891

24. Song Z, Chen W, Athavale D, Ge X, Desert R, Das S, Han H, Nieto N. Osteopontin takes center stage in chronic liver disease. Hepatology. 2021;73:1594-1608

25. Sahai A, Malladi P, Melin-Aldana H, Green RM, Whitington PF. Upregulation of osteopontin expression is involved in the development of nonalcoholic steatohepatitis in a dietary murine model. American journal of physiology. Gastrointestinal and liver physiology. 2004;287:G264-273

26. Lu R, Misra V. Zhangfei: A second cellular protein interacts with herpes simplex virus accessory factor hcf in a manner similar to luman and vp16. Nucleic Acids Research. 2000;28:2446-2454

27. Cui A, Ding D, Li Y. Regulation of hepatic metabolism and cell growth by the atf/creb family of transcription factors. Diabetes. 2021;70:653-664

28. Misra V, Rapin N, Akhova O, Bainbridge M, Korchinski $P$. Zhangfei is a potent and specific inhibitor of the host cell factor-binding transcription factor luman. Journal of Biological Chemistry. 2005;280:15257-15266

29. Zhang F, Hu Z, Li G, Huo S, Ma F, Cui A, Xue Y, Han Y, Gong Q, Gao J, Bian H, Meng Z, Wu H, Long G, Tan Y, Zhang Y, Lin X, Gao X, Xu A, Li Y. Hepatic crebzf couples insulin to lipogenesis by inhibiting insig activity and contributes to hepatic steatosis in diet-induced insulin-resistant mice. Hepatology. 2018;68:1361-1375

30. Hu Z, Han Y, Liu Y, Zhao Z, Ma F, Cui A, Zhang F, Liu Z, Xue Y, Bai J, Wu H, Bian H, Chin YE, Yu Y, Meng Z, Wang H, Liu Y, Fan J, Gao X, Chen Y, Li Y. Crebzf as a key regulator of stat3 pathway in the control of liver regeneration in mice. Hepatology. 2020;71:1421-1436

31. Cui A, Li J, Ji S, Ma F, Wang G, Xue Y, Liu Z, Gao J, Han J, Tai P, Wang T, Chen J, Ma X, Li Y. The effects of b1344, a novel fibroblast growth factor 21 analog, on nonalcoholic steatohepatitis in nonhuman primates. Diabetes. 2020;69:1611-1623 
32. Lin Q, Huang Z, Cai G, Fan X, Yan X, Liu Z, Zhao Z, Li J, Li J, Shi H, Kong M, Zheng M-H, Conklin DJ, Epstein PN, Wintergerst KA, Mohammadi M, Cai L, Li X, Li Y, Tan Y. Activating adenosine monophosphate-activated protein kinase mediates fibroblast growth factor 1 protection from nonalcoholic fatty liver disease in mice. Hepatology. 2021;73:2206-2222

33. Gong Q, Hu Z, Zhang F, Cui A, Chen X, Jiang H, Gao J, Chen X, Han Y, Liang Q, Ye D, Shi L, Chin YE, Wang Y, Xiao H, Guo F, Liu Y, Zang M, Xu A, Li Y. Fibroblast growth factor 21 improves hepatic insulin sensitivity by inhibiting mammalian target of rapamycin complex 1 in mice. Hepatology. 2016;64:425-438

34. Clapper JR, Hendricks MD, Gu G, Wittmer C, Dolman CS, Herich J, Athanacio J, Villescaz C, Ghosh SS, Heilig JS, Lowe C, Roth JD. Diet-induced mouse model of fatty liver disease and nonalcoholic steatohepatitis reflecting clinical disease progression and methods of assessment. American Journal of Physiology-Gastrointestinal and Liver Physiology. 2013;305:G483-G495

35. Wright JF, Le T, Prado J, Bahr-Davidson J, Smith PH, Zhen Z, Sommer JM, Pierce GF, Qu G. Identification of factors that contribute to recombinant aav2 particle aggregation and methods to prevent its occurrence during vector purification and formulation. Molecular Therapy. 2005;12:171178

36. Sommer JüM, Smith PH, Parthasarathy S, Isaacs J, Vijay S, Kieran J, Powell SK, McClelland A, Wright JF. Quantification of adeno-associated virus particles and empty capsids by optical density measurement. Molecular Therapy. 2003;7:122-128

37. Bruha R, Vitek L, Smid V. Osteopontin - a potential biomarker of advanced liver disease. Annals of Hepatology. 2020;19:344-352

38. Syn W-K, Agboola KM, Swiderska M, Michelotti GA, Liaskou E, Pang H, Xie G, Philips G, Chan IS, Karaca GF, Pereira TdA, Chen Y, Mi Z, Kuo PC, Choi SS, Guy CD, Abdelmalek MF, Diehl AM. Nktassociated hedgehog and osteopontin drive fibrogenesis in non-alcoholic fatty liver disease. Gut. 2012;61:1323-1329

39. Guo L, Zhang P, Chen Z, Xia H, Li S, Zhang Y, Kobberup S, Zou W, Lin JD. Hepatic neuregulin 4 signaling defines an endocrine checkpoint for steatosis-to-nash progression. The Journal of clinical investigation. 2017;127:4449-4461

40. Pritchett J, Harvey E, Athwal V, Berry A, Rowe C, Oakley F, Moles A, Mann DA, Bobola N, Sharrocks AD, Thomson BJ, Zaitoun AM, Irving WL, Guha IN, Hanley NA, Hanley KP. Osteopontin is a novel downstream target of sox 9 with diagnostic implications for progression of liver fibrosis in humans. Hepatology. 2012;56:1108-1116

41. Shen $Q$, Christakos $S$. The vitamin d receptor, runx2, and the notch signaling pathway cooperate in the transcriptional regulation of osteopontin. The Journal of biological chemistry. 2005;280:4058940598

42. Agarwal V, Bell GW, Nam J-W, Bartel DP. Predicting effective microrna target sites in mammalian mrnas. eLife. 2015;4:e05005

43. Lin Z, Tian X-Y, Huang X-X, He L-L, Xu F. Microrna-186 inhibition of pi3k-akt pathway via spp1 inhibits chondrocyte apoptosis in mice with osteoarthritis. Journal of Cellular Physiology. 2019;234:6042-6053

44. Afonso MB, Rodrigues PM, Carvalho T, Caridade M, Borralho P, Cortez-Pinto H, Castro RE, Rodrigues CM. Necroptosis is a key pathogenic event in human and experimental murine models of non-alcoholic steatohepatitis. Clinical science (London, England : 1979). 2015;129:721-739

45. Wang X, He Y, Mackowiak B, Gao B. Micrornas as regulators, biomarkers and therapeutic targets in liver diseases. Gut. 2021;70:784-795

46. Wong RJ, Aguilar M, Cheung R, Perumpail RB, Harrison SA, Younossi ZM, Ahmed A. Nonalcoholic steatohepatitis is the second leading etiology of liver disease among adults awaiting liver transplantation in the united states. Gastroenterology. 2015;148:547-555 
Fig. 1

Ma F, et al.

A

A
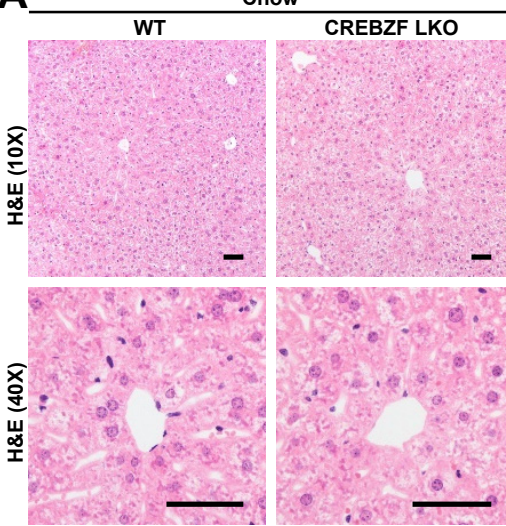

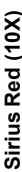

B

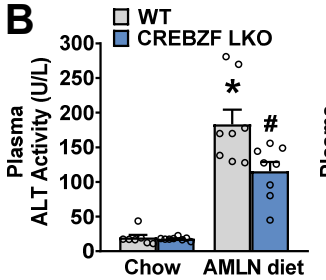

\section{D}

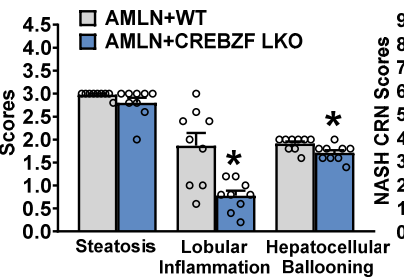

4.5
4.0
3.5
3.0

|
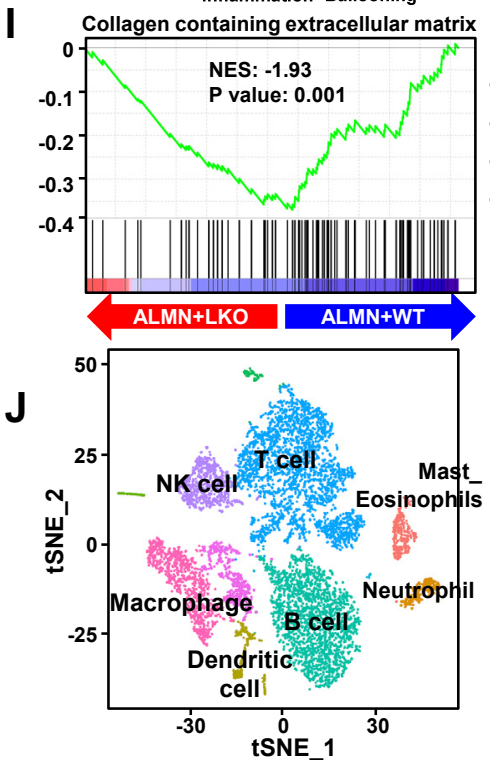

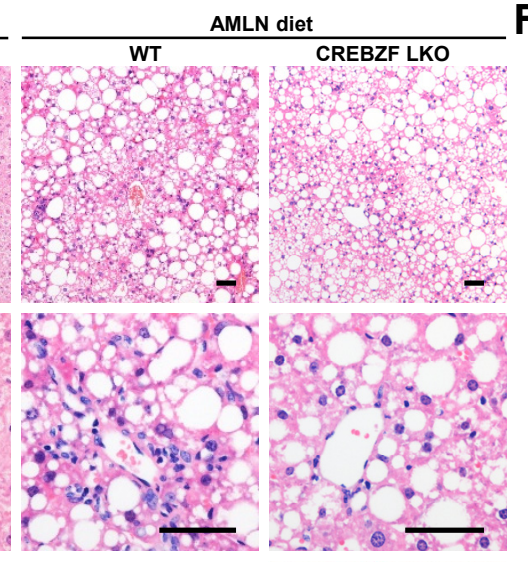

$\mathbf{F}$

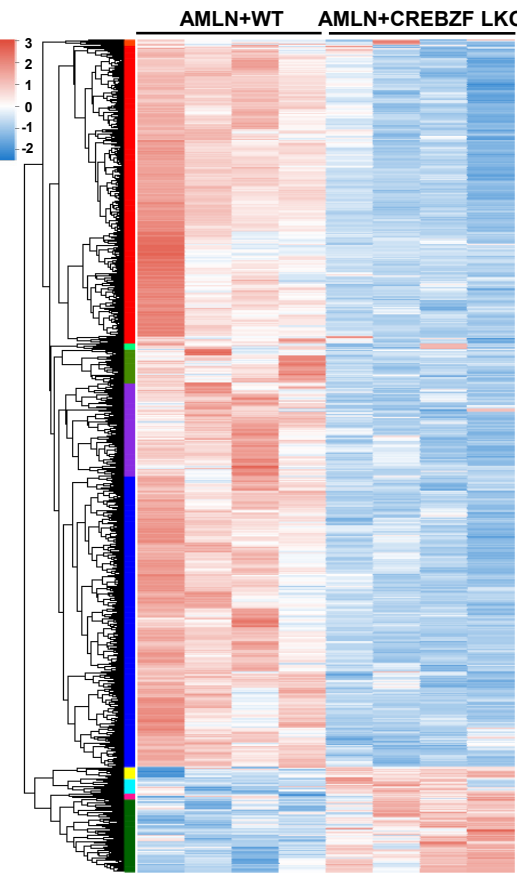

C 2.5 品 WTEBZF LKO
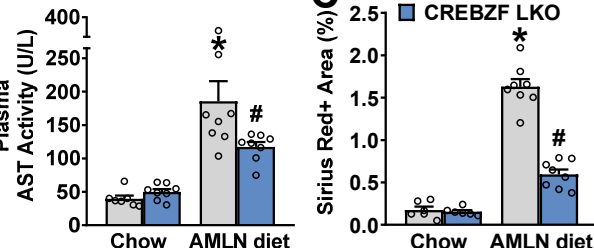

G

KEGG enrichment analysis Number of genes

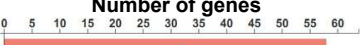

ECM-receptor interaction

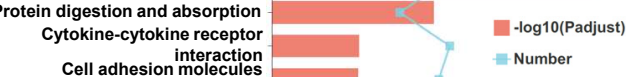

9.0
0.0 .7

$\left.\begin{array}{lll}9.0 & \\ 8.0 & \text { E } & 4.0 \\ 3.5\end{array}\right]$

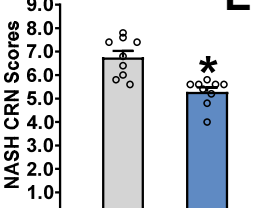

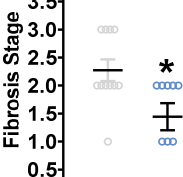

(CAMs)
PI3K-Akt signaling pathway

-

Insulin secretion

Steroid hormone biosynthesis

Chemokine signaling pathway

Inflammatory mediator
regulation of TRP channels

log10 (Padjust)

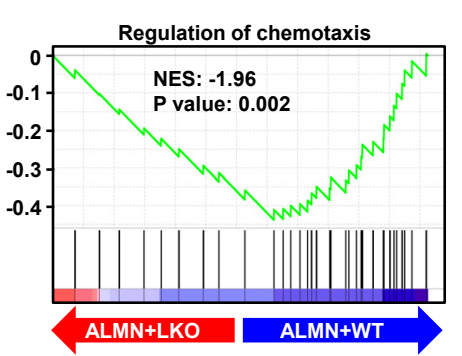

H elastic fiber assembly

GO enrichment analysis

extracellular matrix assembly
regulation of macrophage cytokine production
collagen-activated signaling

pathway

cell-matrix adhesion
extracellular matrix

integrin organization

pathway

pathway
regulation of chemotaxis

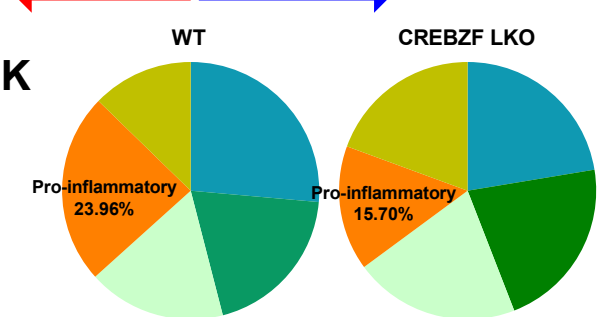

- $26.41 \%$ Cluster 0

- $22.40 \%$ Cluster 0

$19.56 \%$ Cluster 1

$21.69 \%$ Cluster 1

$17.36 \%$ Cluster 2

$\begin{array}{ll}\mathbf{2 0 . 8 1} \% & \text { Cluster } 2 \\ \mathbf{1 9 . 4 0} \% & \text { Cluster } 4\end{array}$

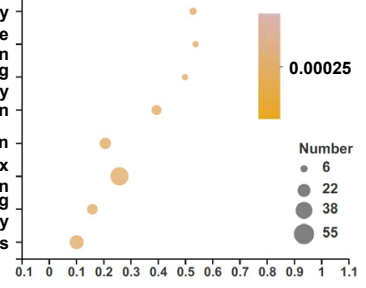

Rich factor

L

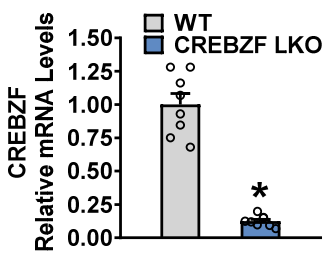


Fig. 2

Ma F, et al.

A

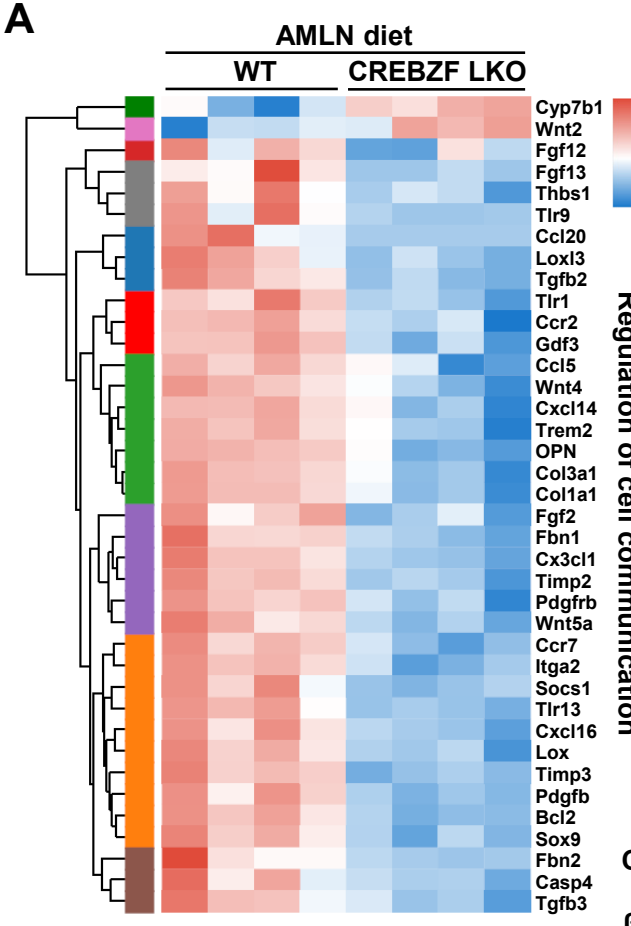

B
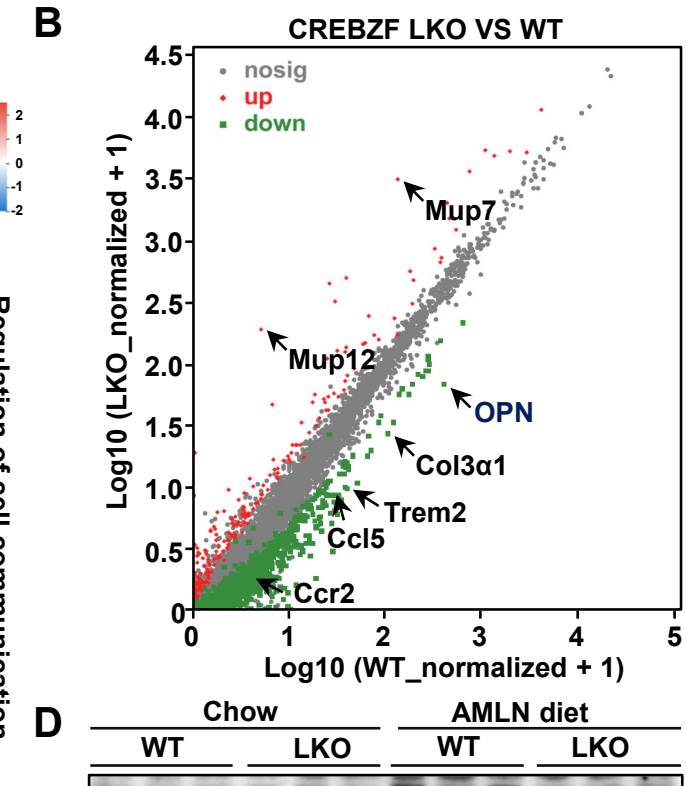

OPN Col1a1

$\beta$-actin $-2-\infty-\infty$

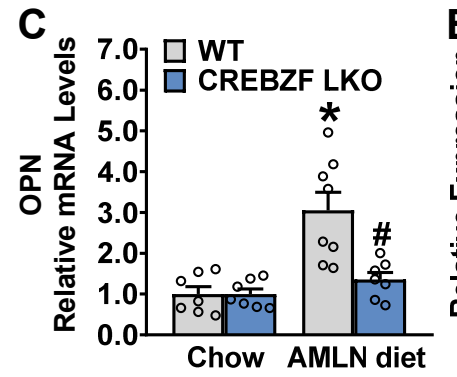

$\mathbf{F}$

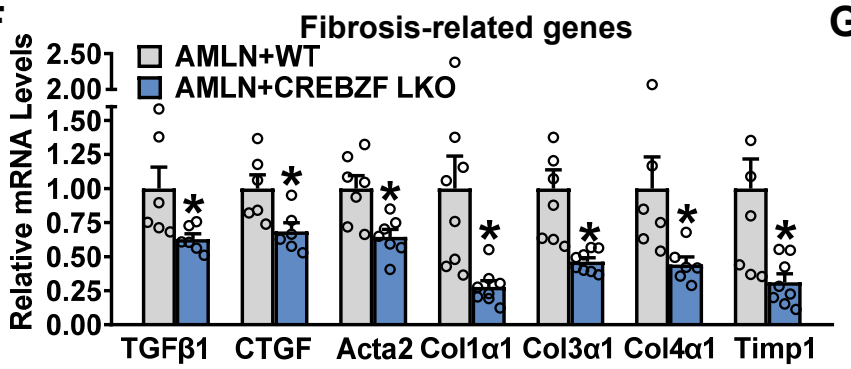

E $5.0 \square$ WT
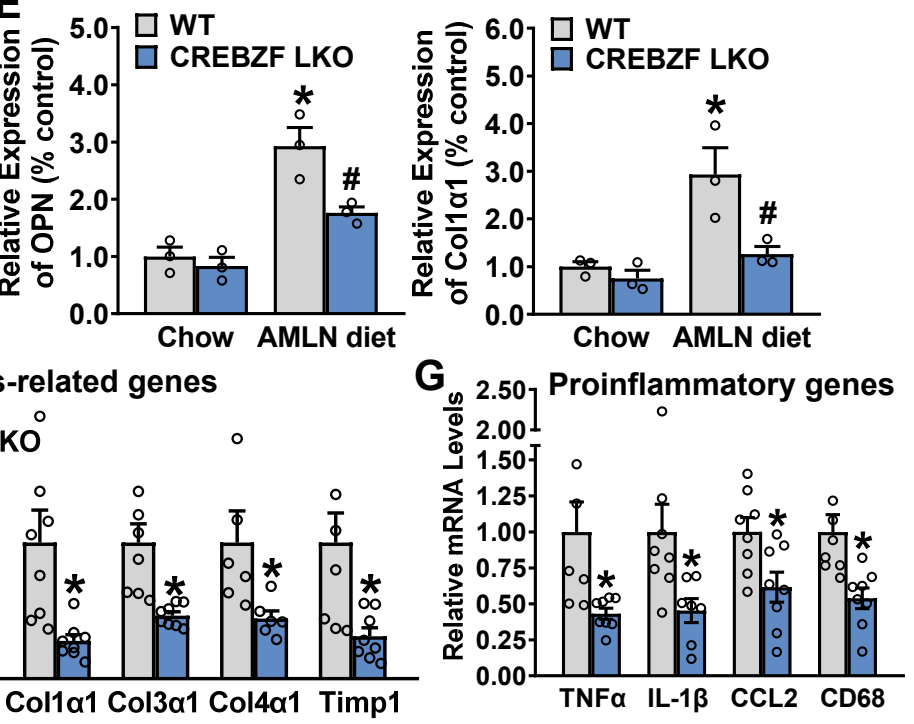
A
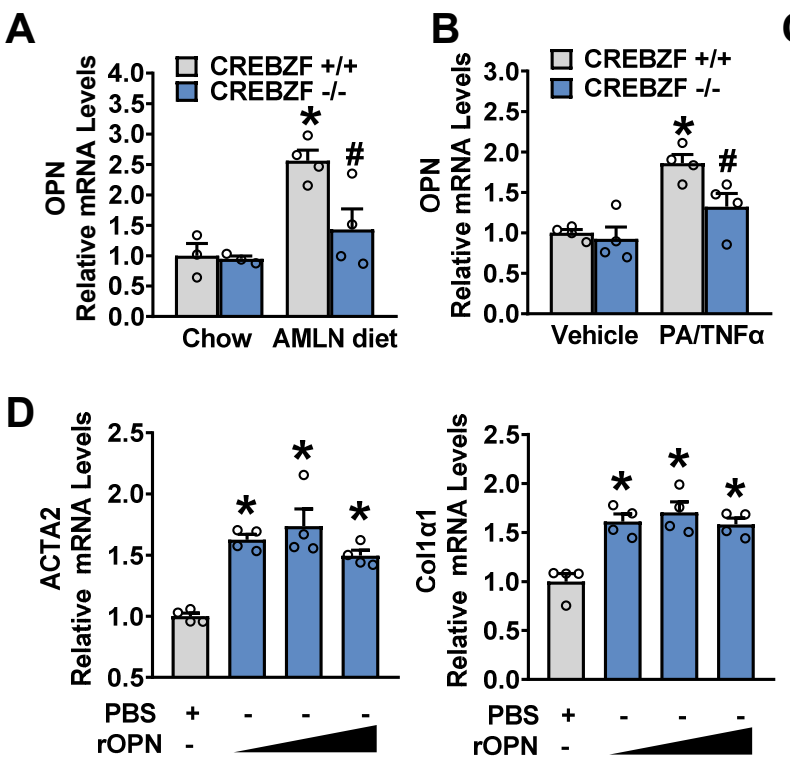

E

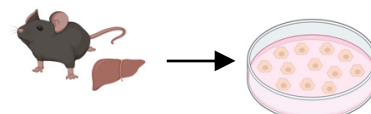

Isolate mouse

PA/TNFa primary hepatocytes treatment

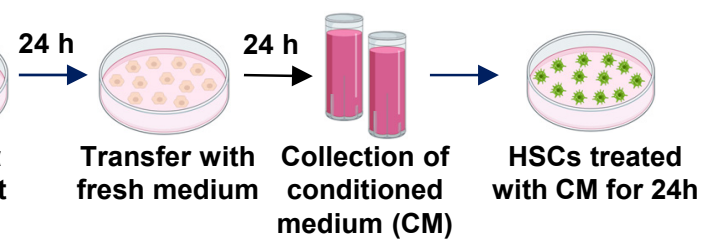

RAW 264.7

$\mathbf{G}$

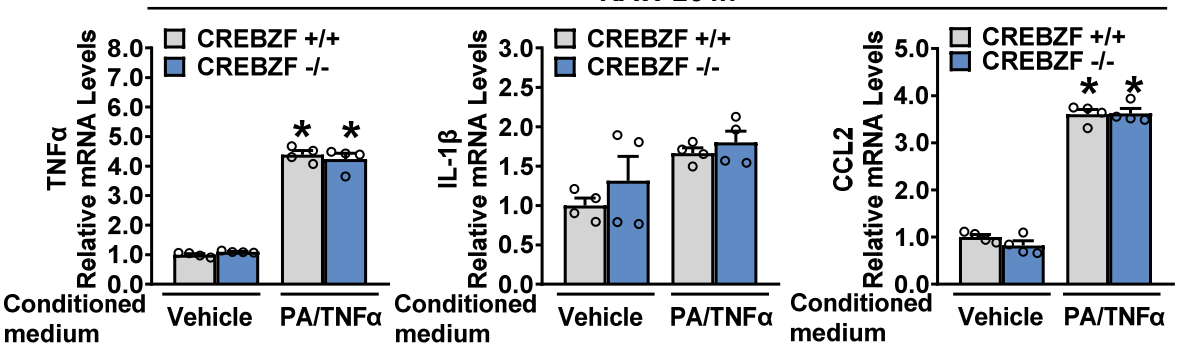

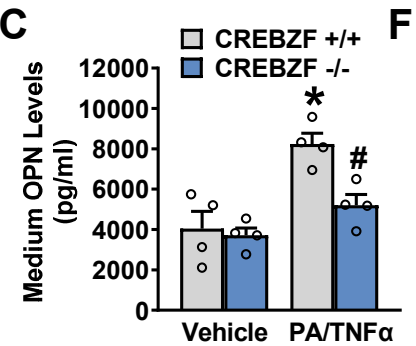
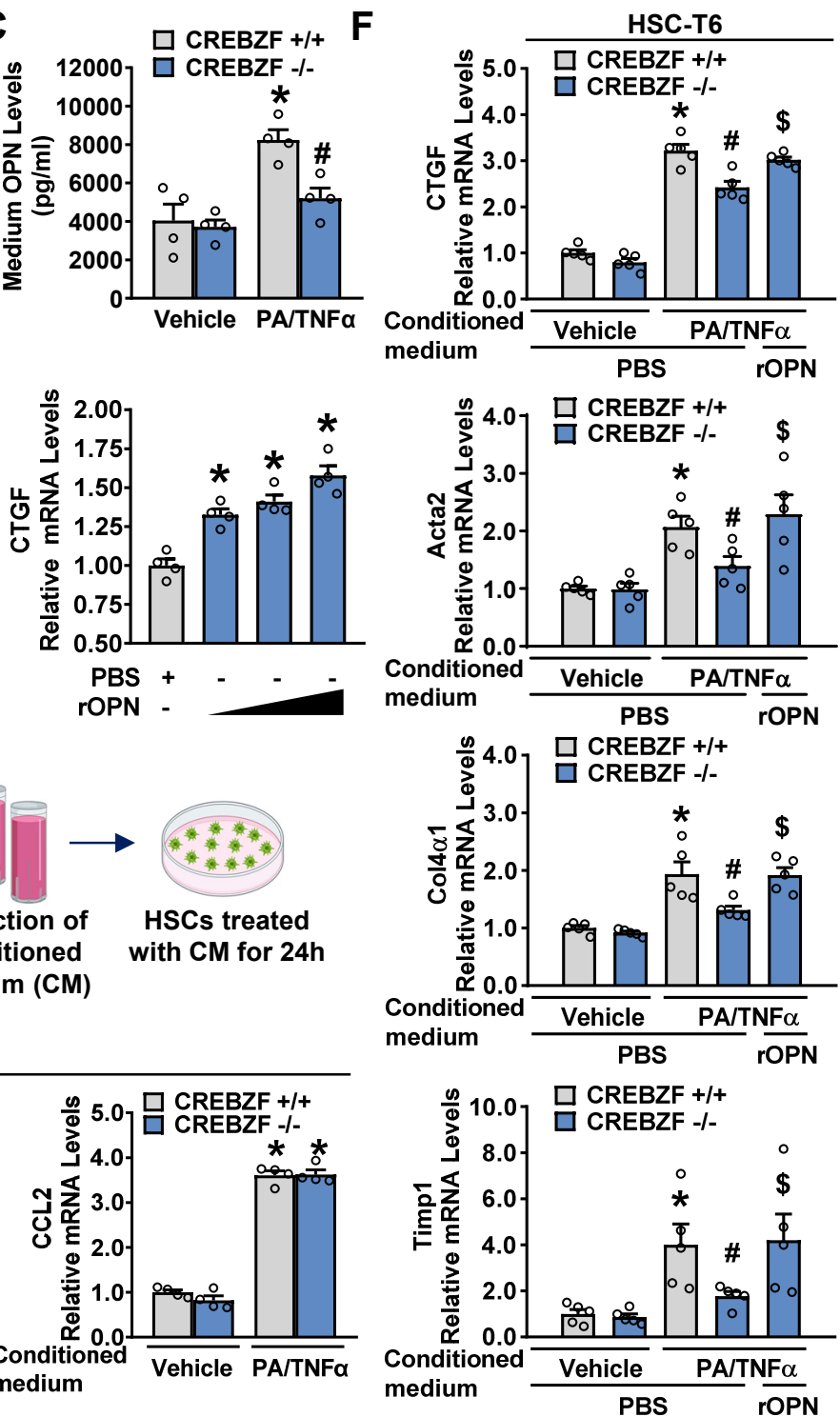
Fig. 4

Ma F, et al.

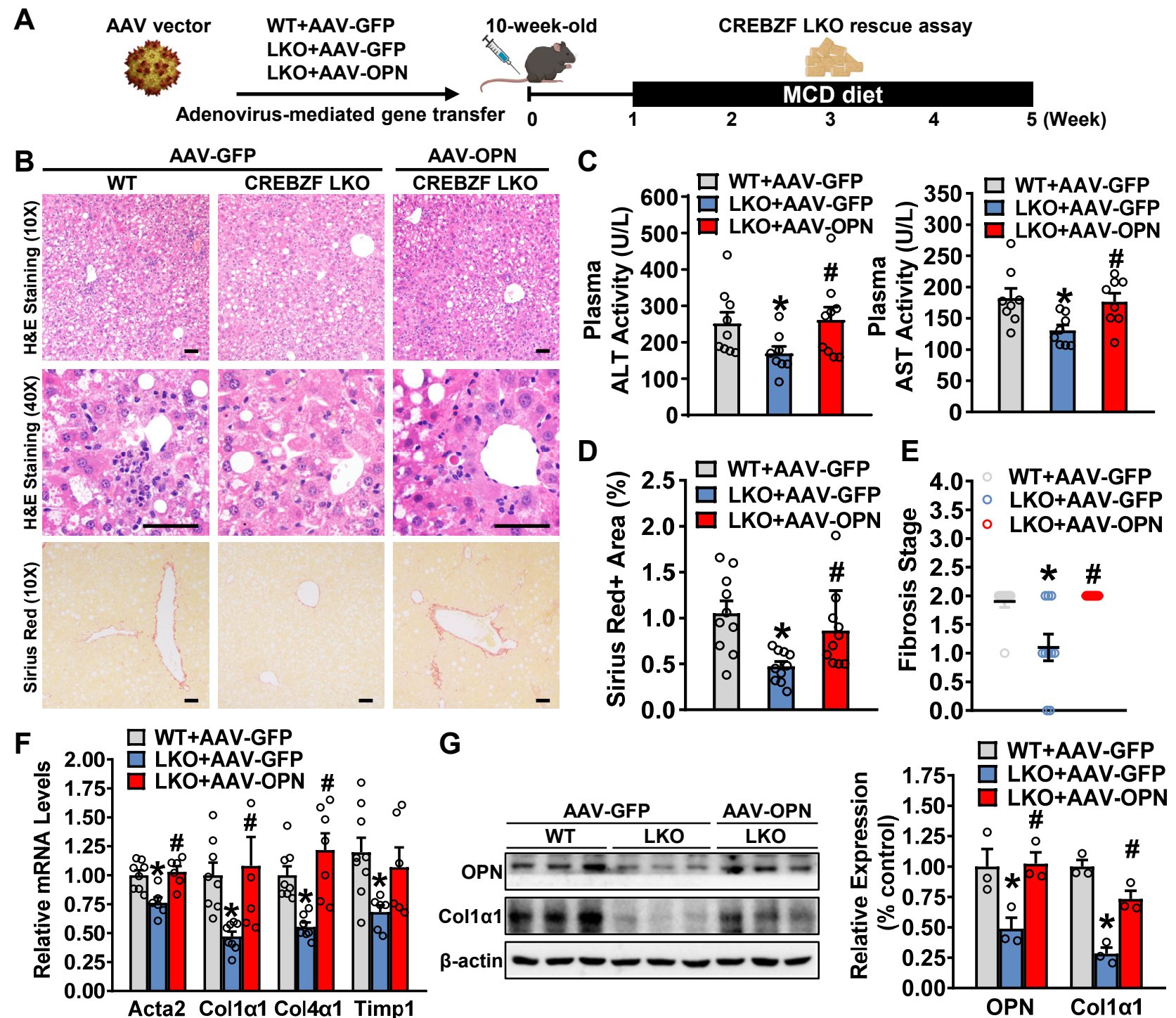


A

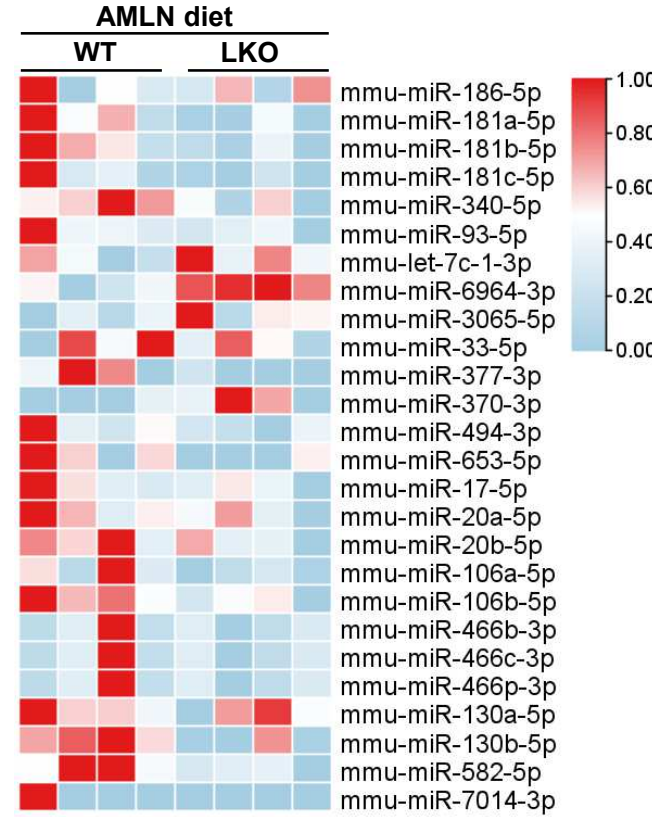

B

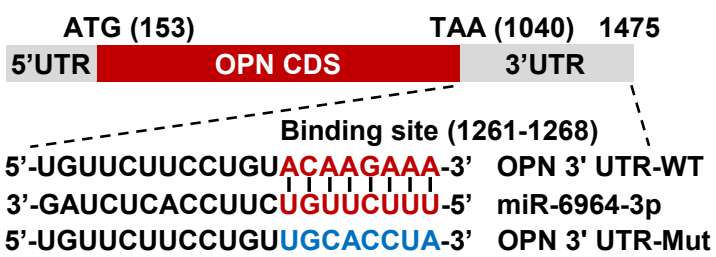

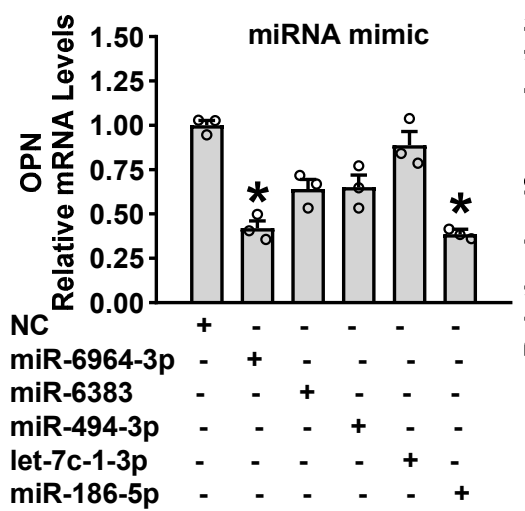

E

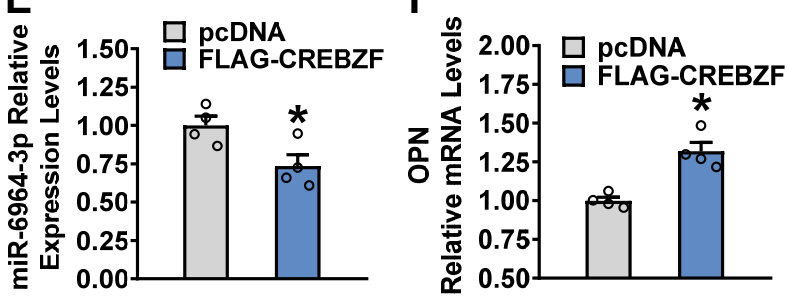

F

C

D
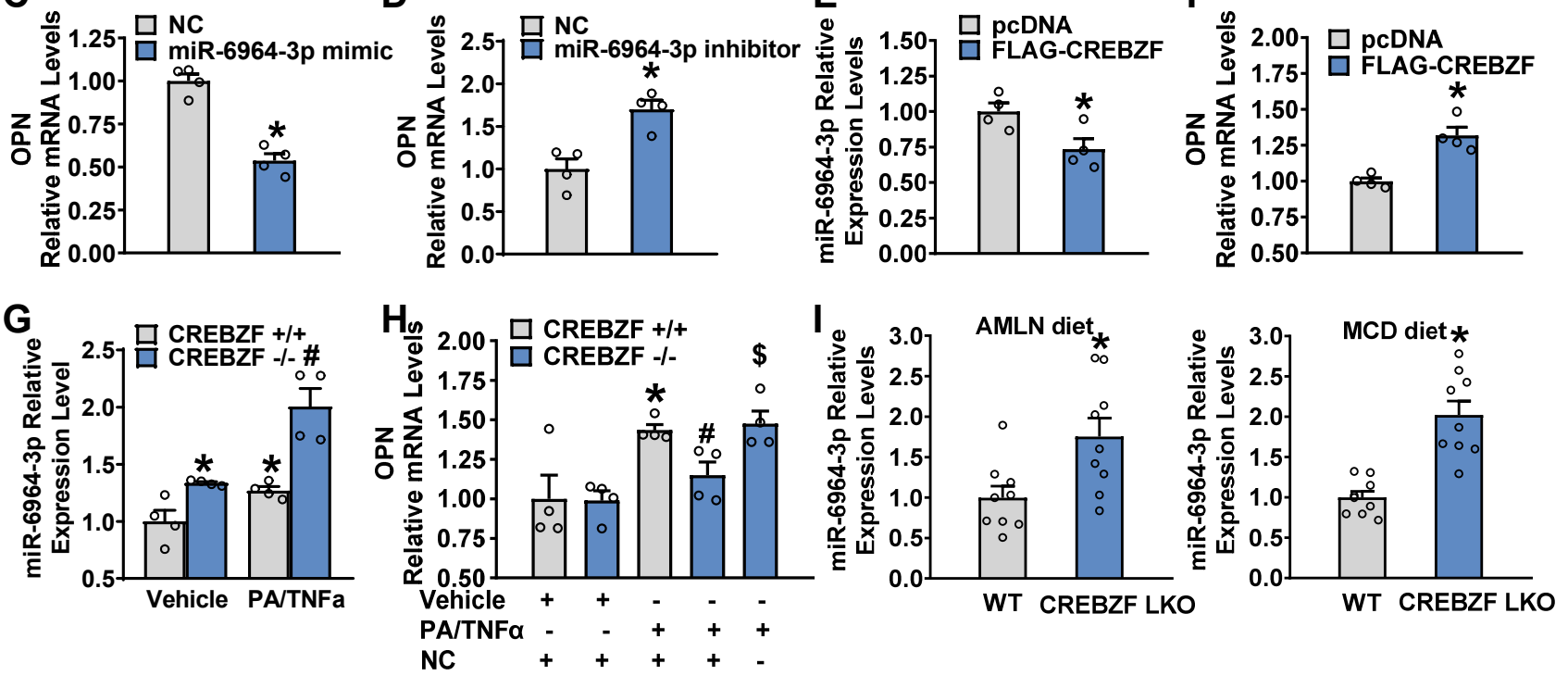

miR-6964-3p inhibitor 


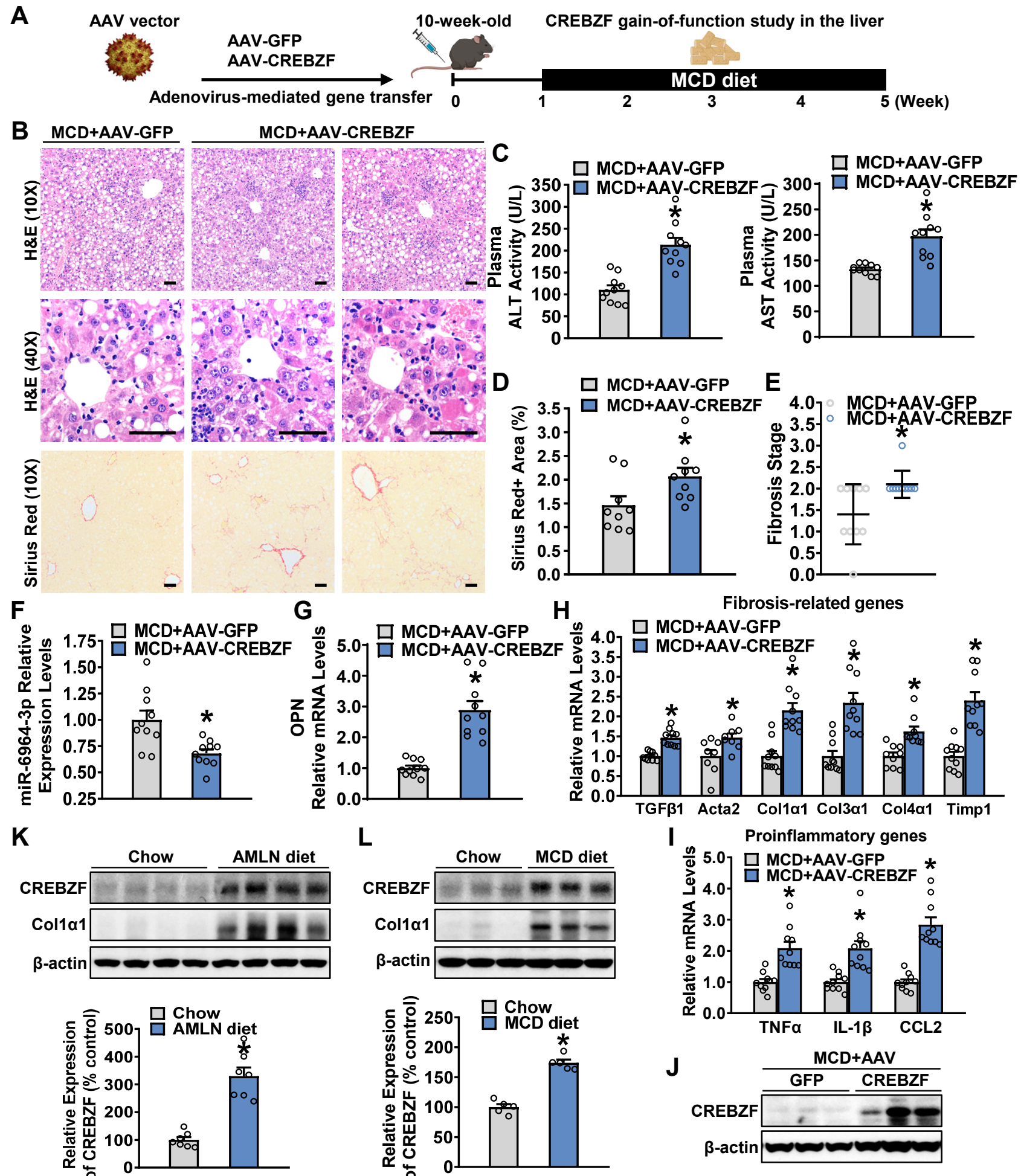


Fig. 7

Ma F, et al.

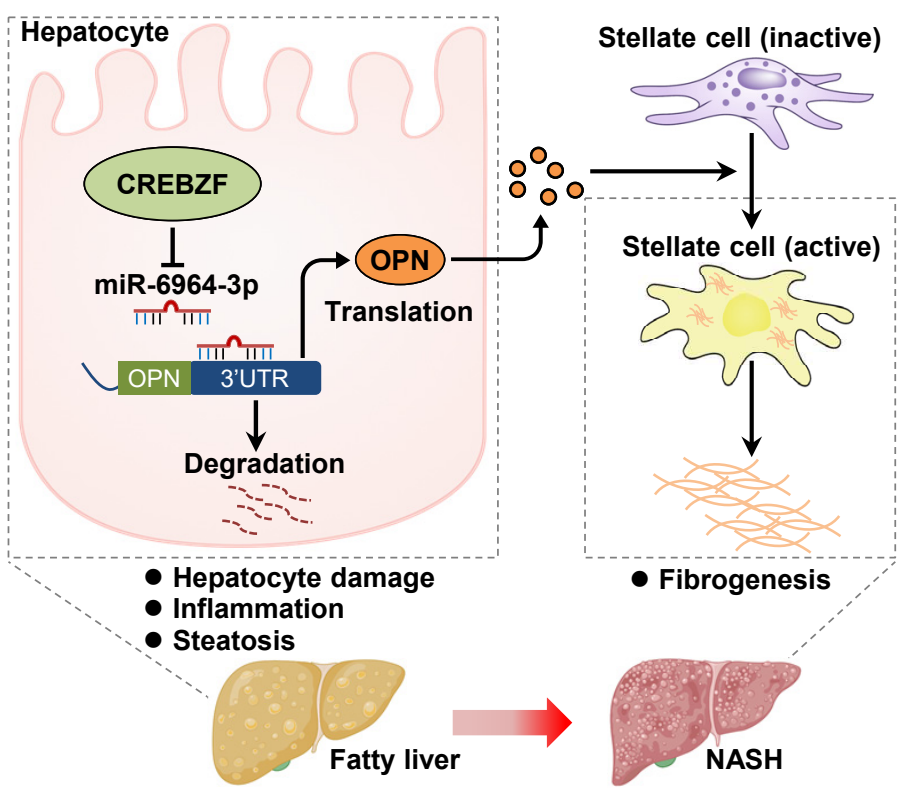




\section{Supplementary Files}

This is a list of supplementary files associated with this preprint. Click to download.

- 3SupplementalTextXFigures.pdf 